\title{
Comparative proteomic analysis of Neisseria meningitidis wildtype and $d p r A$ null mutant strains links DNA processing to pilus biogenesis
}

\author{
Getachew Tesfaye Beyene ${ }^{1,4}$, Shewit Kalayou ${ }^{2,3}$, Tahira Riaz ${ }^{1}$ and Tone Tonjum ${ }^{1,2^{*}}$ (1)
}

\begin{abstract}
Background: DNA processing chain A (DprA) is a DNA binding protein which is ubiquitous in bacteria, and is required for DNA transformation to various extents among bacterial species. However, the interaction of DprA with competence and recombination proteins is poorly understood. Therefore, the proteomes of whole Neisseria meningitidis (Nm) wildtype and dprA mutant cells were compared. Such a comparative proteomic analysis increases our understanding of the interactions of DprA with other $\mathrm{Nm}$ components and may elucidate its potential role beyond DNA processing in transformation.

Results: Using label-free quantitative proteomics, a total of 1057 unique Nm proteins were identified, out of which 100 were quantified as differentially abundant $(P \leq 0.05$ and fold change $\geq|2|)$ in the dprA null mutant. Proteins involved in homologous recombination (RecA, UvrD and HolA), pilus biogenesis (PilG, PilT1, PilT2, PilM, PilO, PilQ, PilF and PilE), cell division, including core energy metabolism, and response to oxidative stress were downregulated in the Nm dprA null mutant. The mass spectrometry data are available via ProteomeXchange with identifier PXD006121. Immunoblotting and co-immunoprecipitation were employed to validate the association of DprA with PilG. The analysis revealed reduced amounts of PilG in the dprA null mutant and reduced amounts of DprA in the $\mathrm{Nm}$ pilG null mutant. Moreover, a number of pilus biogenesis proteins were shown to interact with DprA and /or PilG.
\end{abstract}

Conclusions: DprA interacts with proteins essential for Nm DNA recombination in transformation, pilus biogenesis, and other functions associated with the inner membrane. Inverse downregulation of Nm DprA and PilG expression in the corresponding mutants indicates a link between DNA processing and pilus biogenesis.

Keywords: Neisseria meningitidis, DNA processing, Pilus biogenesis, DprA, PilG, Proteomics, Mass spectrometry

\section{Background}

Neisseria meningitidis (Nm) is a human commensal and pathogen that in the lack of bactericidal antibodies may cause meningitis and/or septicaemia [1]. Nm has a small $(\sim 2.2 \mathrm{Mb})$ and hyperdynamic genome. The pathogenic Neisseria species, $\mathrm{Nm}$ and $\mathrm{N}$. gonorrhoeae $(\mathrm{Ng})$ are naturally and constitutively competent for uptake of exogenous DNA provided that they express type 4 pili

\footnotetext{
* Correspondence: tone.tonjum@medisin.uio.no

${ }^{1}$ Department of Microbiology, University of Oslo, Oslo, Norway

2Department of Microbiology, Oslo University Hospital, Oslo, Norway

Full list of author information is available at the end of the article
}

(Tfp), can perform RecA-dependent recombination, and find the abundantly occurring DNA uptake sequence (DUS) in the transforming DNA [2-4]. Transformation is the main form of horizontal gene transfer (HGT) in Neisseria $s p$., enabling these species to generate extensive genetic diversity $[5,6]$.

During transformation, the incoming DNA is processed by RecA [7, 8], DNA processing chain A (DprA), recombination mediator protein (RMP), and singlestranded DNA-binding protein (SSB) [4, 9-13]. The association of RecA with DNA is mainly its central role in homologous recombination [14]. DprA from Streptococcus 
pneumoniae, Bacillus subtilis, and Helicobacter pylori was shown to take part in intracellular DNA processing, interact with RecA, and displace SSB from ssDNA $[15,16]$. In addition, DprA loads RecA onto ssDNA, promoting annealing of homologous ssDNA, and protects incoming DNA [15-18]. The DprA and RecA proteins bind strongly and in long clusters to ssDNA to form a nucleoprotein filament $[15,17]$. DprA selectively binds and protects ssDNA from nucleases [8]. DprA plays a role in transformation in all bacterial species examined except for Escherichia coli; however, the transformability of $d p r A$ null mutants varies among bacterial species and DNA substrates. Using a transposon mutant screen in Nm, Tang and co-workers showed that the $\operatorname{dpr} A$ null mutant exhibits total loss of competence for DNA transformation [4]. The $\mathrm{Nm}$ and Ng dprA null mutants are nontransformable regardless of the type of donor DNA substrate, and Ng DprA is suggested to be involved in RecA-mediated pilin variation [4, 19]. HGT in Haemophilus influenzae, S. pneumoniae, and B. subtilis is associated with DprA [8, 15, 20, 21]. DprA in S. pneumoniae is involved in an intracellular signalling cascade that turns off competence [22, 23]. DprA in B. subtilis appears to increase the efficiency of RecA strand exchange during transformation and forms a large multiprotein complex with RecA, SSB-B and other competence proteins $[17,24]$.

Here, we performed a comprehensive proteomic analysis of Nm wildtype and $d p r A$ null mutant cells to define their protein profile and to search for interactions between DprA and other Nm components. For this purpose, the cell lysates from the Nm wildtype and $d p r A$ null mutant strains were prepared, and the proteins were subjected to in-gel digestion. The resulting peptide products were subsequently analysed by using high resolution mass spectrometry (MS).

In this global quantitative proteomic analysis, multiple proteins identified were significantly less abundant in the $d p r A$ null mutant including those involved in Tfp biogenesis, recombination, cell division and energy metabolism. A link between DprA and the inner membrane protein PilG and other pilus biogenesis proteins was thereby detected. Immunoblotting and coimmunoprecipitation (Co-IP) were employed to validate the interaction between DprA and PilG. In general, these findings elucidate the role of DprA in Nm cells and its interaction with components of the transformation, Tfp biogenesis, and other machineries.

\section{Results}

Predominantly less abundant proteins detected in the $\mathrm{Nm}$ $\Delta d p r A$ mutant

To assess DprA-associated changes in the Nm proteome, a quantitative analysis of $\mathrm{Nm}$ wildtype and $d p r A$ mutant strains was conducted by applying a liquid chromatography tandem mass spectrometry (LC-MS/ MS)-based label free quantitative (LFQ) proteomics approach. Total soluble lysate from three biological replicates were separated by one dimensional (1D) SDS-PAGE. After tryptic in-gel digestion, six gel fractions from each replicate were analyzed by high performance liquid chromatography (HPLC) coupled with Q Exactive MS in technical triplicates. This workflow generated a total of 108 Raw MS files. The resulting data was analyzed together in the MaxQuant environment specifying a confidence rate of $99 \%$ at the peptide and protein level. This identified a total of 1057 protein groups, with 1010 proteins identified in the wildtype, and 915 proteins identified in the DprA null mutant (Fig. 1a, Additional file 1: Table S1). Analysis for overlap of protein identification showed an overlap of $70.7 \%(647 / 915)$ and $77.6 \%(784 / 1010)$ protein identification across all the biological experiments in the $d p r A$ mutant and wildtype, respectively (Fig. 1a-c). Likewise, the overall overlap between $\operatorname{dpr} A \mathrm{mu}$ tant and wildtype samples was 82\% (868/1057). In order to check the reproducibility of our label-free quantification workflow, we computed the Pearson correlations ( $\mathrm{R}$ values) of biological replicates using normalized protein LFQ intensities. The analysis showed that the $R$ values (range 0.67-0.96) between normalized intensities were high and was thus suited for accurate comparisons of protein abundance differences.

For comparative analysis, protein groups with at least two valid LFQ values out of three biological replicates were considered. This criterion yielded a total of 1028 protein group eligible for quantitative analysis. Using a combination of $p \leq 0.05$ and a fold change cutoff of \pm 2 , the analysis resulted in 100 proteins whose abundances were significantly changed (Fig. 2; Additional file 1: Table S1). Unsupervised hierarchical clustering (HC) of these significantly changed proteins showed that the LFQ values profile of $d p r A$ null mutant proteins clustered together distinctly from the wildtype protein samples (Fig. 3). Among the differentially abundant (DA) proteins, $16 \%$ were upregulated, and $84 \%$ were downregulated in the $\mathrm{Nm} d p r A$ null mutant, respectively (Fig. 2; Additional file 1: Table S1).

\section{DprA affects proteins involved in recombination / 3R functions}

The distribution of the Nm DA proteins enriched by the Kyoto Encyclopedia of Genes and Genomes (KEGG) category is shown in Fig. 4a and b. Most of the DA proteins belonged to the KEGG functional categories of genetic information processing, energyand amino acid- metabolism, and signaling and 

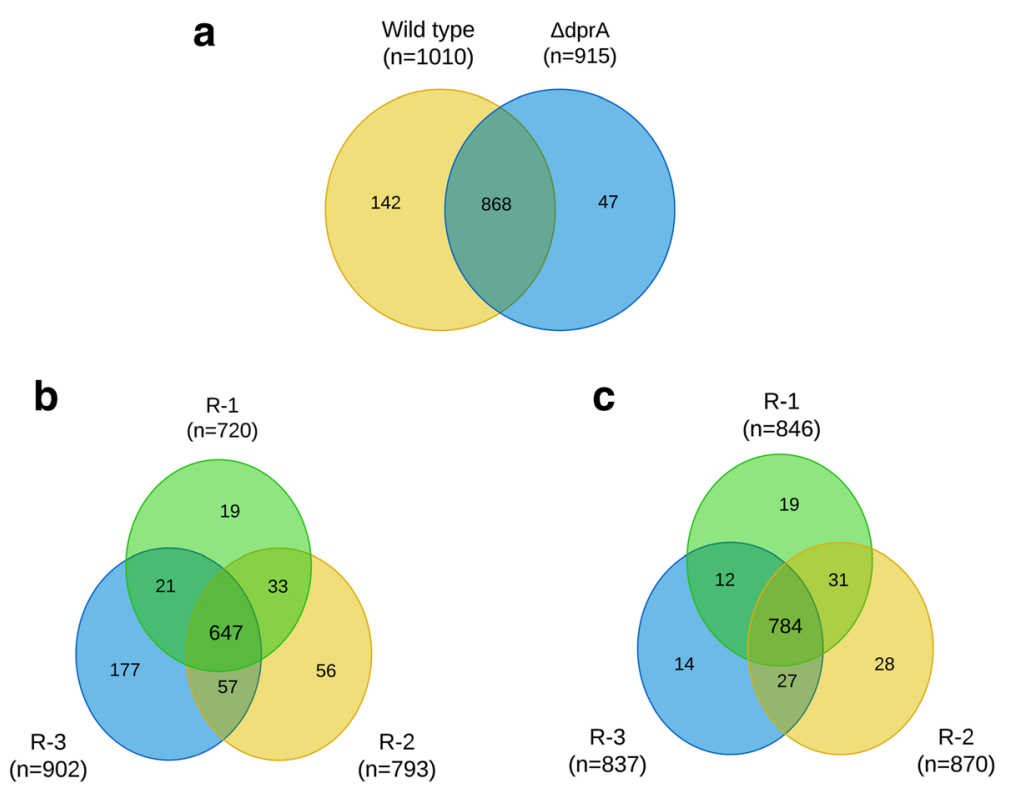

Fig. 1 Venn plot illustrating protein identification overlaps between wildtype and $\triangle d p r A(\mathbf{a})$ and among the three biological replicates (R1-R3) in $\triangle$ dprA mutant (b) and Wildtype (c)

cellular process. The genetic information processing proteins constitutes the DNA repair, recombination and replication (3R) proteins (Fig. 4). Notably, the RecA, UvrD, and HolA proteins (Table 1) which play roles in replication and homologous recombination were significantly downregulated in the $\mathrm{Nm} d p r A$ null mutant relative to the wildtype. In addition, several of the $3 R$ proteins including the replicative helicases RuvAB and DnaB, the DNA polymerase III (Pol III) $\beta$ and $\varepsilon$ subunits, DNA topoisomerase I, and DNA gyrase subunits $\mathrm{A}$ and $\mathrm{B}$, as well as DNA translocase FtsK1 and FtsK2 were detected less abundant in the DprA mutant relative the wild type strain. However, the differences detected in the replication proteins were not significant. On the other hand, the single strand binding protein (SSB), DNA-binding protein (HupB), and the DNA Pol III $\gamma$ and $\tau$ subunits were moderately more DA in the DprA mutant. This upregulation was not significant, yet detectable (Table 1).

DprA influences proteins involved in pilus biogenesis and twitching motility

A number of proteins involved in pilus biogenesis and twitching motility, the PilG, PilM, PilT1 and PilT2 (Table 2) were differentially less abundant in the $\mathrm{Nm}$ dprA null mutant relative to the wildtype strain. Notably, by MS analysis the amounts of PilG were 50X reduced in the DprA mutant. Conversely, DprA was 0.6X downregulated in the $\mathrm{Nm} \Delta$ pilG mutant by MS analysis (Tables 2 and 3). Although not significantly regulated, the pilus assembly protein PilF, PilO, and the twitching motility protein (PilU, NMB0051) were found to be less abundant in the Nm DprA mutant, whereas PilE, PilQ, and the putative fimbrial biogenesis and twitching motility protein NMB1309 were slightly more abundant (Table 2).

\section{Proteins involved in core metabolism are significantly regulated by DprA}

The majority of proteins involved in cell division, carbohydrate- and energy-metabolism (e.g, the oxidoreductases), and amino acid metabolism were significantly regulated. For example, the cell division proteins FtsA (NMB0426), FtsZ (NMB0427), ZipA (NMB0667), MinD (NMB0171), and Lon (NMB1231) were downregulated when DprA was lacking. Also, the ATP-binding cassette (ABC transporters, NMB0387, NMB1240, and NMB1226) were significantly less abundant in the $\mathrm{Nm} d p r A$ mutant, whereas the amino acid $\mathrm{ABC}$ transporter and the permease protein NMB1509 were significantly more abundant (Table 4). The Nm core metabolism components Pgm, $\mathrm{CbbA}$, and TpiA of the glycolysis, NADH-quinone oxidoreductases NuoI (NMB0251), Mqo (NMB2096), Icd of the tricarboxylic acid (TCA) cycle, and the enzyme which catalyze the subpathway of leucine biosynthesis LeuA were downregulated in the lack of DprA. On the other hand some proteins involved in glycolysis (GapA-2), TCA cycle (NqrB), and amino acid metabolism (ArgF and $\mathrm{ArgH}$ ) were upregulated in $\mathrm{Nm} d p r A$ mutant (Table 4). 


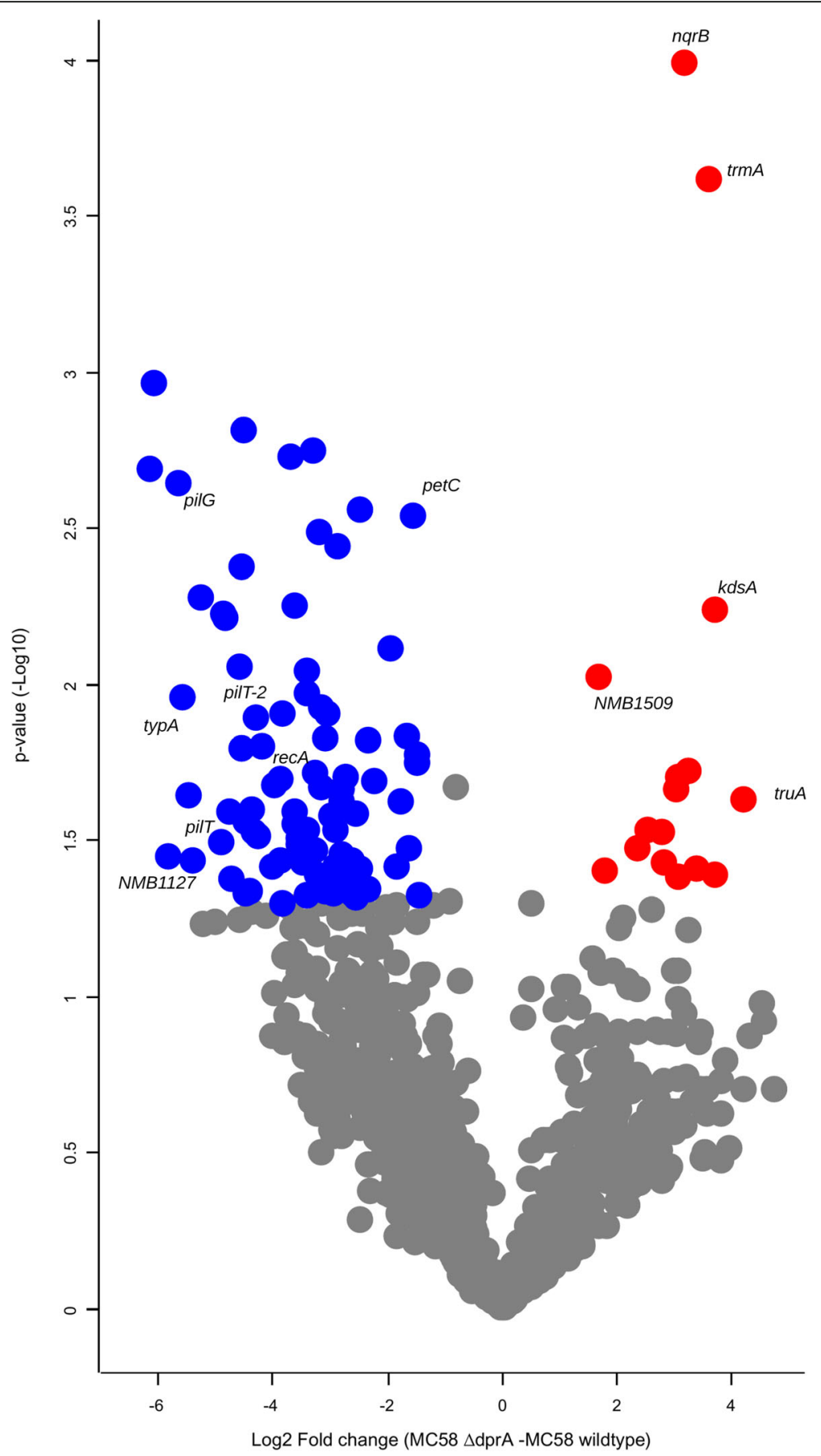

Fig. 2 Quantitative analysis of differential protein abundances in Nm serotype B. Volcano plot of protein abundance differences as a function of statistical significance (t test $p \leq 0.05$ and fold change cutoff $\geq 2$ ) between $d p r A$ null mutant and wildtype Nm. More abundant proteins are indicated by blue dots and less abundant proteins by red dots

Defense against oxidative stress is affected by DprA

$\mathrm{Nm}$ inhabits the oxygen-rich niche made up by the human oral mucosa and inevitably encounters continuous damage from the exposure to reactive oxygen species (ROS) [25]. Hence, multiple membrane proteins that are relevant in $\mathrm{Nm}$ pathogenesis and defense against oxidative stress proteins such as the Kat (Catalase), TrxB (thioredoxin reductase), and the c-type cytochromes [Cytochrome c (NMB0923), Cytochrome c5 (NMB1677), and the ubiquinol-cytochrome $\mathrm{c}$ reductase, cytochrome c1 (PetC)] were less abundant in the $d p r A$ null mutant (Table 4). 

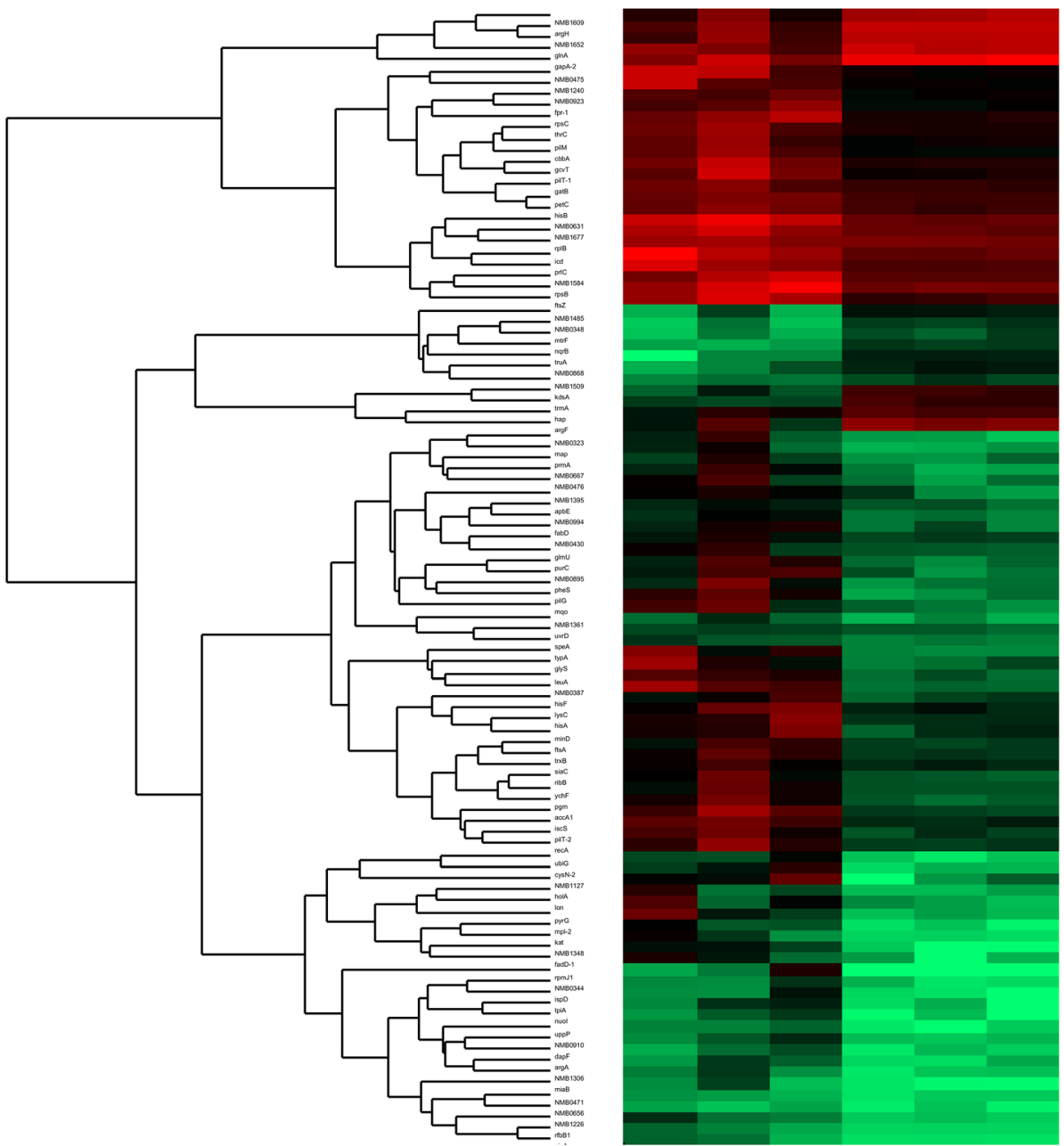

Fig. 3 Unsupervised hierarchical clustering of LFQ values of 100 significantly abundant proteins $(p \leq 0.05$, fold change $=2$ ) in $d p r A$ null mutant relative to the wildtype $\mathrm{Nm}$. Samples with similar pattern of intensities of the significant proteins are clustered while dendrograms indicate cluster produced by Euclidean distance of proteins. Red and green colors indicate higher and lower abundances, respectively

\section{DprA networks of proteins associated with the inner membrane}

To define the functional and molecular interaction network, all the DA proteins were searched for in the online STRING protein query database. Eighty-one out of the 100 DA proteins exhibited evidence to be present in recognized and predicted networks with a total interaction edges of 147 (Fig. 5). This functional interaction
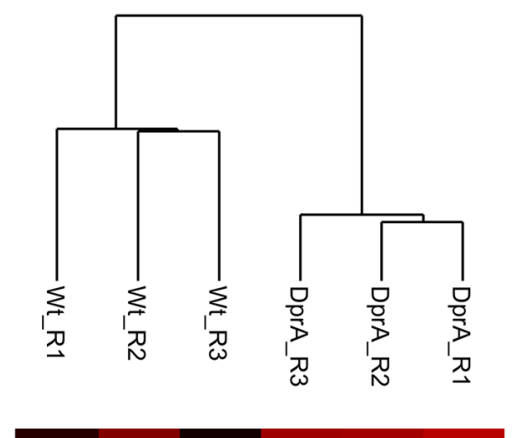

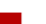



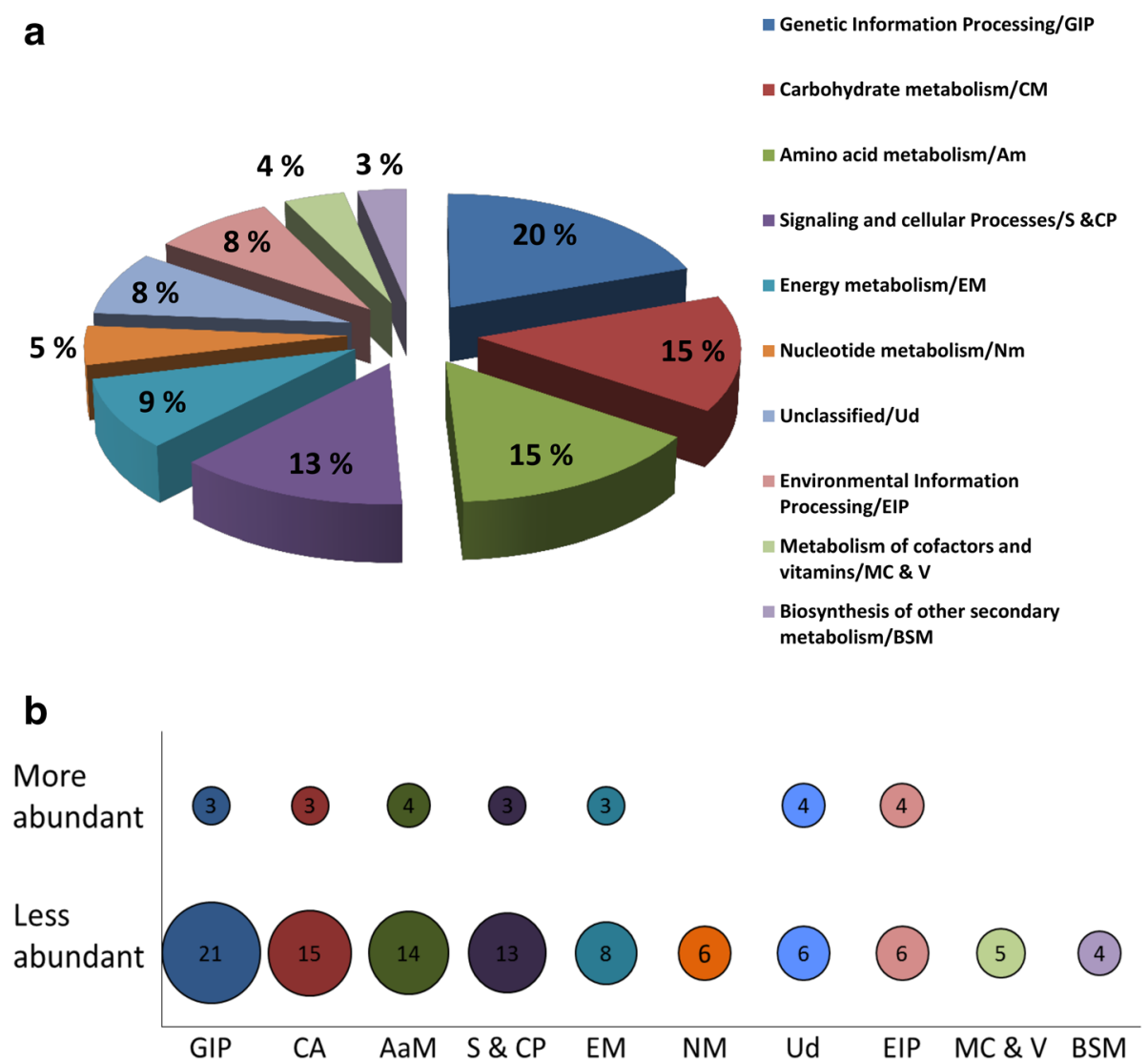

Fig. 4 Function category of significantly differentially abundant (DA) proteins in $\triangle d p r A$ mutant and the wild type Nm. A total of $100 \mathrm{DA}$ protein sequences retrieved from UniProtKB were used as query dataset input and uploaded to BlastKOALA (KEGG Orthology and Links Annotation). The taxonomic group of genome, and the KEGG genes database file search used in the analysis were Prokaryotes:Bacteria, and species_prokaryotes.pep respectively. Approximately $92 \%$ of the DA proteins were functionally annotated (a). A bubble plot comparison of differentially less- and moreabundant proteins, the bubble size is proportional to the counts proteins (b). GIP - Genetic Information Processing; CM - Carbohydrate metabolism, AaM - Amino acid metabolism, S \& CP - Signalling and cellular Processes, EM - Energy metabolism, NM - Nucleotide metabolism, Ud - Unclassified, EIP - Environmental Information Processing, MCV - Metabolism of cofactors and vitamins, and BSM - Biosynthesis of other secondary metabolism

\section{DprA and PilG mutants exhibit mutual downregulation}

By MS analysis, reduced amounts of PilG were detected in the $d p r A$ null mutant, and reduced amounts of DprA protein were detected in the Nm pilG null mutant. In order to validate the data obtained by MS analysis, we assayed the relative abundance of the DprA and PilG proteins by immunoblotting (Fig. 6, Additional file 2: Figure S2). The results from immunoblotting show that the amounts of these two proteins were mutually reduced in the null mutants. The level of PilG expression detected by immunoblotting was reduced in $d p r A$ null mutant $\mathrm{Nm}$; however, the reduction of the level of expression was not significant. DprA levels were also reduced in the PilG null mutant Nm (Fig. 6). Further pull-down analysis by Co-IP coupled to MS analysis and immunoblotting revealed that most of the pilus biogenesis proteins that were detected, and/or identified less abundant in $\Delta d p r A$ mutant in global proteome analysis, were found interact with DprA (Table 5, Additional file 3: Figure S3 A). The cell division proteins
MinD, FtsZ, and FtsA which were significantly less abundant in the $\Delta d p r A$ mutant, were found to interact with DprA. In addition, the recombinational repair proteins RecO, RecR, SSB, and TopA were detected as DprAinteracting partner proteins. Both PilG and PilE revealed interactions with PilT, PilN and SSB (Table 5). The detailed lists of the interacting partner proteins of DprA, PilG, or PilE are found in the Additional file 4 (Table S2). By MS analysis, the abundance of PilE in the $\triangle d p r A$ mutant was shown to be equivalent to that of the abundance of PilE in the wildtype Nm, which was also confirmed by immunoblotting (Additional file 3: Figure S3 b, c). In the $\triangle$ pilG mutant, the lack of PilE and the presence of S-pilin were confirmed [26].

\section{Discussion}

DprA in bacteria is so far shown to be required for competence for DNA transformation [4] and suggested to be involved in RecA-mediated pilin variation [19]. The presence 
Table 1 The $3 R$ (replication, recombination and repair) gene products that were differentially abundant in the Nm $\triangle d p r A$ mutant compared to the wildtype

\begin{tabular}{|c|c|c|}
\hline Genes & Protein name/function & Protein fold change \\
\hline$\overline{h o l} A^{\mathrm{a}}$ & DNA polymerase III $\delta$-subunit & -22.66 \\
\hline$r e c A^{a}$ & Recombinase protein & -14.67 \\
\hline ftsk2 & DNA translocase & -13.05 \\
\hline recN & DNA repair protein & -12.33 \\
\hline gyrA & DNA gyrase subunit $A$ & -11.68 \\
\hline muts & DNA mismatch repair protein & -11.59 \\
\hline topA & DNA topoisomerase 1 & -10.19 \\
\hline$d n a Q-2$ & DNA polymerase III $\varepsilon$-subunit & -7.30 \\
\hline$d n a B$ & Replicative DNA helicase & -4.75 \\
\hline$d n a N$ & DNA polymerase III $\beta$-subunit & -4.59 \\
\hline rmuC & DNA recombination protein RmuC homolog & -4.15 \\
\hline $\operatorname{ruv} B$ & ATP-dependent DNA helicase & -3.68 \\
\hline gyrB & DNA gyrase subunit $B$ & -2.33 \\
\hline$u v r D^{a}$ & DNA helicase & -1.78 \\
\hline ftsK1 & DNA translocase & -1.60 \\
\hline rdgC & Recombination-associated protein & -1.52 \\
\hline polA & DNA polymerase I & -1.48 \\
\hline ruva & ATP-dependent DNA helicase & -1.47 \\
\hline dnax & DNA polymerase III subunits $\gamma$ and $\tau$ & +2.07 \\
\hline$s s b$ & Single-stranded DNA-binding protein & +2.37 \\
\hline hupB & DNA-binding protein $\mathrm{HU}-\beta$ & +4.27 \\
\hline
\end{tabular}

${ }^{a}$ Significantly regulated proteins. The minus sign of the protein fold change indicates the downregulated whereas the positive sign indicates upregulated proteins

Table 2 Gene products involved in transformation and pilus biogenesis that were differentially abundant in the Nm $\triangle d p r A$ mutant compared to the wildtype when identified by mass spectrometry

\begin{tabular}{llc}
\hline Genes & Protein name & Protein fold change \\
\hline pilG $^{\text {a }}$ & Pilus assembly protein PilG & -50.33 \\
pilT-2 $^{\mathrm{a}}$ & Twitching motility protein PilT & -14.24 \\
pilT-1 $^{\mathrm{a}}$ & Twitching motility protein PilT & -11.75 \\
pilM $^{\mathrm{a}}$ & PilM protein & -6.24 \\
NMB0051 (pilU) & Twitching motility protein & -3.24 \\
pilF & Type IV pilus assembly protein & -2.88 \\
pilO & PilO protein & -2.57 \\
pilN & PilN protein & -1.08 \\
NMB0889 (pilX) & pilus assembly protein & -1.05 \\
NMB0888 (pilW) & Putative type IV pilus assembly protein & +1.07 \\
pilP & PilP protein & +1.39 \\
pilQ & Type IV pilus biogenesis and competence protein PilQ & +1.50 \\
NMB1309 & Putative fimbrial biogenesis and twitching motility protein & +1.54 \\
pilE & Fimbrial & +1.61 \\
\hline
\end{tabular}

${ }^{a}$ Significantly downregulated proteins in the $\Delta d p r A$ mutant. The minus sign of the protein fold change indicates the downregulated whereas the positive sign upregulated proteins 
Table 3 LFQ values for DprA in Neisseria meningitidis wildtype and $\triangle$ pilG mutant detected by mass spectrometry

\begin{tabular}{llll}
\hline Nm strain & DprA LFQ1 & DprA LFQ2 & DprA LFQ3 \\
\hline Wildtype & 3420300 & 1884100 & 2652200 \\
Nm $\triangle$ pilG & 1244200 & 1823000 & 1533600 \\
\hline
\end{tabular}

of a $d p r A$ gene has been suggested to be a distinctive feature of naturally transformable species [27]. DprA is ubiquitous in the microbial kingdom and its orthologs exist in certain eukaryotes, suggesting more fundamental functions for DprA. In one study, dprA was indicated to be one of the essential genes of $\mathrm{Nm}$ to cause invasive disease in an infant rat model [28]. However, beyond that, evidence of functions of DprA beyond transformation particularly its interaction with the pilus biogenesis and other proteins by Co-IP and proteome analysis has not formerly been presented. Here, we demonstrate that proteins involved in homologous recombination during transformation (RecA, UvrD and HolA), pilus biogenesis (PilG, PilM, PilT1, PilT2, PilE, PilQ, PilF, and PilO), and cell division, as well as core energy metabolism, and response to oxidative stress were less abundant in $\mathrm{Nm} d p r A$ null cells (Fig. 5). Inverse downregulation of Nm DprA and PilG expression in the corresponding mutants indicate a link between DprA and the inner membrane protein PilG that could be attributed to either DNA processing or pilus biogenesis (Fig. 6). Notably, pull-down experiments using Co-Coupled to MS analysis demonstrated that DprA interacted with the pilus biogenesis protein PilG.

Genetic compensation where cells respond to the reduced expression of a gene by up-regulating compensatory genes/pathways is a well-known process $[29,30]$. In this case, $\mathrm{Nm}$ responded to the loss of $d p r A$ expression by DA of a number of proteins involved in processes as diverse as recombinational-repair and replication, pilus

Table 4 Selected gene products significantly differentially abundant proteins in the Nm $\triangle d p r A$ mutant compared to the wildtype when identified by mass spectrometry

\begin{tabular}{|c|c|c|}
\hline Genes & Protein name/function & $\begin{array}{l}\text { Protein fold } \\
\text { change }\end{array}$ \\
\hline \multicolumn{3}{|c|}{ Cell division proteins } \\
\hline $\mathrm{fts} A$ & Cell cycle & -7 \\
\hline ZipA & cell septum assembly & -24 \\
\hline lon & Lon protease/cell cycle & -22 \\
\hline $\mathrm{fts} Z$ & Cell cycle & -12 \\
\hline $\min D$ & Septum site-determining protein & -11 \\
\hline \multicolumn{3}{|c|}{ Proteins of core metabolism } \\
\hline \multicolumn{3}{|c|}{ Glycolysis and TCA cycle } \\
\hline tpiA & Fructose and mannose metabolism; Glycolysis & -20.72 \\
\hline pgm & Amino sugar and nucleotide sugar metabolism & -19.52 \\
\hline$c b b A$ & Fructose and mannose metabolism; Glycolysis & -10.60 \\
\hline icd & Carbon fixation; Citrate cycle (TCA cycle) & -8.59 \\
\hline gapA-2 & Glyceraldehyde-3-phosphate dehydrogenase & 5.70 \\
\hline $\operatorname{argH}$ & Arginine and proline metabolism & 8.34 \\
\hline $\arg F$ & Arginine and proline metabolism & 12.90 \\
\hline \multicolumn{3}{|c|}{ Electron transport chain } \\
\hline NMB1127 & Oxidoreductase, short chain dehydrogenase & -56 \\
\hline mao & Probable malate:quinone oxidoreductase & -27 \\
\hline nuol & NADH-quinone oxidoreductase subunit I & -16 \\
\hline$n q r B$ & $\mathrm{Na}(+)$-translocating $\mathrm{NADH}$-quinone reductase subunit $\mathrm{B}$ & +9 \\
\hline NMB0994 & Acyl-CoA dehydrogenase family protein & -7 \\
\hline NMB0923 & Cytochrome c & -6 \\
\hline NMB1677 & Cytochrome c5 & -5 \\
\hline petC & Ubiquinol cytochrome $\mathrm{c}$ reductase & -3 \\
\hline kat & Catalase & -22 \\
\hline $\operatorname{trx} B$ & Thioredoxin reductase & -8 \\
\hline
\end{tabular}

The minus sign of the protein fold change indicates the less abundant whereas the positive sign denotes the more abundant proteins. 


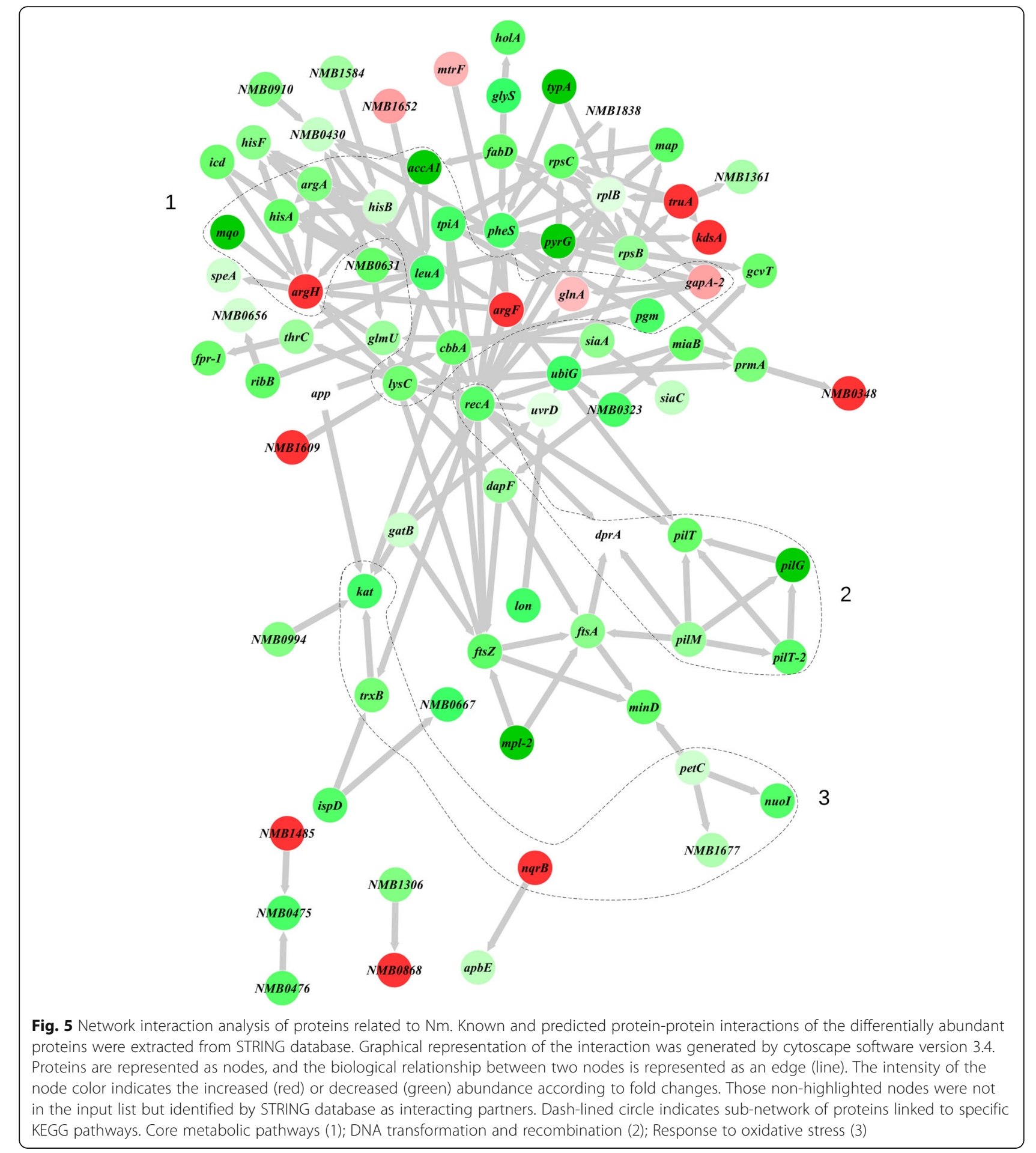

biogenesis and twitching motility, cell division, energy-, and amino acid-metabolism (Fig. 4a and b).

In the $d p r A$ mutant, RecA was less abundant. RecA plays a central role in recombination and recombinational DNA repair [31]. The Holliday junction processing RuvAB [32], UvrD, and HolA were also downregulated proteins of the $3 \mathrm{R}$ genes. UvrD resolves
Holliday junctions and is part of the mismatch and nucleotide excision repair pathways [33, 34]. HolA catalyzes DNA replication as a component of the Polymerase III holoenzyme [35]. However, in contrast to the reduced abundance of many of these $3 \mathrm{R}$ proteins, the survival of $d p r A$ mutant and the wild type $\mathrm{Nm}$ was virtually the same when exposed to genotoxic agents 


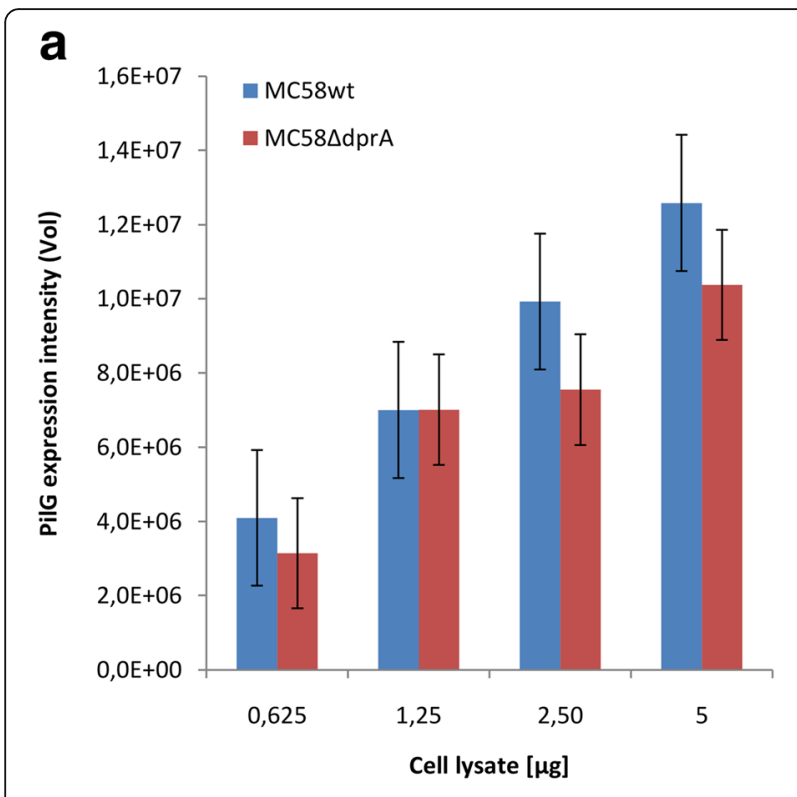

b

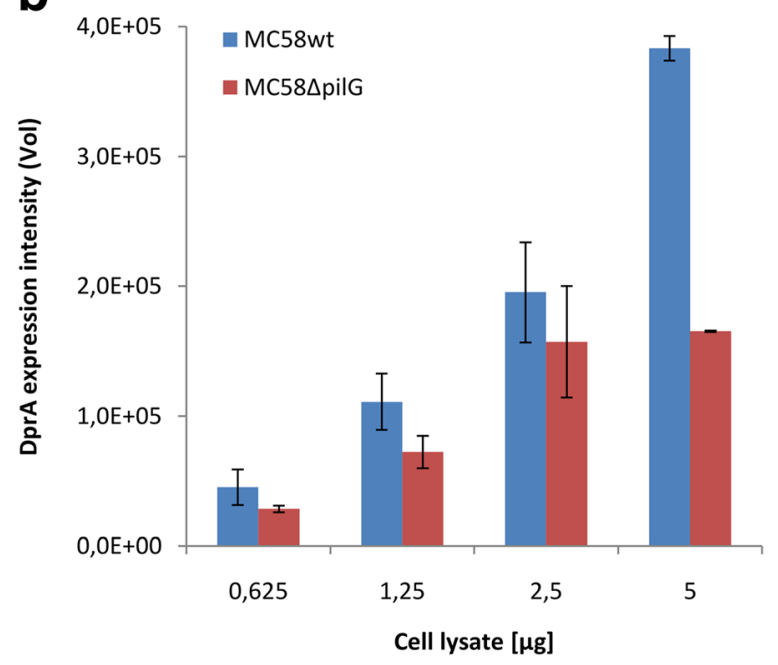

Fig. 6 PilG and DprA immunoblots. MC58 wt and $\triangle$ dprA mutant overnight cultures were harvested in $1 \times$ PBS and heat inactivated at $60^{\circ} \mathrm{C}$ for $30 \mathrm{~min}$. The cells were disrupted using MagNa Lyser (6x 90 s at a speed of $6000 \mathrm{rpm})$. The cell lysates were prepared in Laemmli sample buffer and SDS-PAGE was run. The proteins transferred onto PVDF membrane. The membranes were incubated with ati-PilG (a) and ati-DprA (b) rabbit polyclonal primary antibodies, and anti-rabbit-lgG-horseradish peroxidase conjugate secondary antibody. The immunoblots were developed with Immun-Star WesternC

Chemiluminescent kit (Bio-Rad) and visualized using a ChemiDoc Touch imager (Bio-Rad). Results were analysed using the Image Lab software (Bio-Rad)

[36]. The survival of the $d p r A$ mutant equivalent to the wildtype $\mathrm{Nm}$ also contrasts with the downregulation of FtsK1 and FtsK2 proteins synchronizing chromosome segregation and cell division [37]. Furthermore, topoisomerases controlling the topology of DNA, TopA, GyrA, and GyrB [38, 39], were less abundant without DprA.
The pilus biogenesis proteins were among the less abundant differentially regulated proteins in $\mathrm{Nm} \Delta d p r A$ cells (Table 2; Fig. 6A). An interesting finding with regard to the function of DprA in transformation is that, components of the Tfp biogenesis proteins PilG, PilM, PilT1, and PilT2 proteins were less abundant in the Nm dprA mutant. DprA was also less abundant in the $\mathrm{Nm}$ $\Delta$ pilG mutant, suggesting a potential direct or indirect interaction between these components (Fig. 5). We have previously shown that PilG is absolutely required for $\mathrm{Nm}$ pilus biogenesis [26] and directly binds DNA [40, 41] and the secretin PilQ in transformation [40, 42]. The neisserial pilG and pilT mutants and the Thermus thermophilus pilM mutant are defective for transformation [43-46]. T. thermophilus PilM binds the inner membrane protein PilN, as well as ATP, and its structure is most similar to the actin-like protein FtsA [47]. In addition, PilT has been shown to be important in $\mathrm{Nm}$ pathogenesis and was upregulated in $\mathrm{Nm}$ grown in human blood [48]. The pilus subunit and biogenesis proteins essential for neisserial interaction with host cells and competence for transformation (PilE and PilC) [49, 50], including those required for DNA transformation PilQ, PilF, and PilO [42, 46, 51, 52], were found less abundant in the mutant strain. Nevertheless, the data presented here is not adequate to finally conclude that the synergistic effects of the reduced expression of proteins related to pilus biogenesis and RecA functions in the Nm DprA mutant strain have contributed for the transformation phenotype. $\mathrm{Nm}$ responds to the stress from growing in human blood by metabolic adaptation and energy conservation by downregulating some of the cell division genes, such as $\min D$ and $f t s Z$ [53]. In our study, FtsA, FtsZ, ZipA, MinD, and Lon proteins were significantly less abundant in the lack of $d p r A$. In addition, the direct interaction of $\mathrm{Nm}$ DprA with FtsZ and MinD in vitro was demonstrated. ZipA in association with FtsA facilitates the formation and stabilization of septum (FtsZ-ring) at midcell during cell division [54]. MinD is a membrane-associated ATPase which determines the site of septum formation [55]. Lon is a DNA dependant ATPase involved in cell division and production of capsular polysaccharide [56]. However, despite the reduced abundance of these proteins, the $\mathrm{Nm} d p r A$ mutant exhibited growth phenotypes comparable to the wildtype strain [36]. One of the reasons for this observation could be that the levels of these cell division proteins were not a rate limiting factor in the growth conditions used, or because of other compensatory changes that occurred in the Nm $d p r A$ mutant $[29,30]$.

As a mucosal pathogen and survivor in the blood stream of humans, $\mathrm{Nm}$ needs to possess effective defense mechanisms to survive the oxidative stress produced by cells of the immune system [57]. In this 
Table $\mathbf{5}$ List of proteins identified to interact with DprA or PilG by pull-down experiments with co-immunoprecipitation (Co-IP) and mass spectrometry (MS)

\begin{tabular}{|c|c|c|c|c|c|c|c|}
\hline \multirow[t]{3}{*}{ Protein IDs } & \multirow[t]{3}{*}{ Protein names } & \multirow[t]{3}{*}{ Gene names } & \multicolumn{5}{|c|}{$\begin{array}{l}\text { Co-IP and MS identified proteins that } \\
\text { interact with DprA, PilG, and/or PilE }\end{array}$} \\
\hline & & & \multicolumn{2}{|c|}{ Anti-DprA-Ab } & \multicolumn{2}{|c|}{ Anti-PilG-Ab } & \multirow{2}{*}{$\begin{array}{l}\text { Anti-PilE-Ab } \\
W t\end{array}$} \\
\hline & & & Wt & $\triangle d p r A$ & $\mathrm{Wt}$ & $\Delta p i l G$ & \\
\hline Q9K1K1 & DNA processing chain A & $d p r A$ & + & - & - & - & - \\
\hline Q7DDR1 & Pilus assembly protein & pilG & + & + & + & - & + \\
\hline $\begin{array}{l}\text { P05431 Q4W588 Q4W587 Q4W586 } \\
\text { Q4W589 Q4W585 Q4W590 Q4W592 }\end{array}$ & Fimbrial protein; PilS cassette & pilE \& pils & + & + & + & + & + \\
\hline Q4W563 & PilN protein & pilN & + & + & + & + & + \\
\hline Q70M91 & $\begin{array}{l}\text { Type IV pilus biogenesis and competence } \\
\text { protein PilQ }\end{array}$ & pilQ & + & + & - & + & - \\
\hline Q7DD78 & PilO protein & pilO & - & - & - & + & - \\
\hline Q9JY02 & PilM protein & pilM & - & + & - & - & - \\
\hline Q7DDU1 & Twitching motility protein & pilT-1 & - & - & - & + & + \\
\hline POAOS6 & Cell division protein & $f t s Z$ & + & + & - & - & - \\
\hline Q9K0X8 & Cell division protein & $f t s A$ & - & + & - & - & - \\
\hline Q7DDS7 & Septum site-determining protein & $\min D$ & + & + & - & - & - \\
\hline Q9JXD7 & Probable malate:quinone oxidoreductase & mao & + & + & - & - & - \\
\hline Q7DDL4 & Glutaredoxin & NMB0773 & - & - & - & + & - \\
\hline PODH59 & Protein RecA & rec $A$ & - & + & + & + & - \\
\hline P66849 & Single-stranded DNA-binding protein & ssb & + & + & + & - & + \\
\hline Q9JZ92 & Recombination protein & reck & + & - & - & - & - \\
\hline Q9K0W0 & DNA repair protein & reco & + & - & - & - & - \\
\hline Q9K1K0 & DNA topoisomerase 1 & topA & + & - & - & - & - \\
\hline
\end{tabular}

Most of these proteins were also revealed to be significantly less abundant in the Nm $\triangle d p r A$ mutant as compared to wildtype (wt) by MS of whole cell lysates. In Co-IP, DprA, PilG, or PilE was used as bait proteins through their reactivity with specific rabbit polyclonal antibodies (Ab). In order for DprA, PilG or PilE to target their interacting proteins, the Ab against the bait proteins were incubated with the cell lysates from Nm wt as well as the cell lysates from the Nm $\Delta d p r A$ or $\Delta p i l G$ mutant (in the mutant the bait protein is absent), and thereby the Ab binds the bait protein. The bait protein coupled with the antibody binds its interacting partner, forming the antibody-bait-prey protein complex. The "+" sign designates the formation of antibody-bait-prey protein complex whereas the "-" sign designates the absence of complex formation/interaction

context, the oxidoreductases important in the eukaryotes as well as in the bacterial respiratory chain and defense against oxidative stress [58] were less abundant in the $\mathrm{Nm}$ dprA mutant. For example, the short chain dehydrogenase/reductase (NMB1127) is a member of $\mathrm{NAD}(\mathrm{P})(\mathrm{H})$ dependent oxidoreductases which play critical roles in lipid, amino acid, and carbohydrate metabolisms [59]. The $\mathrm{NADH}$-quinone oxidoreductase NuoI (NMB0251) and Mqo (NMB2096) were also significantly downregulated. These oxidoreductases are primarily the main entry site for electrons to the respiratory chain of aerobic bacteria [60-62]. Particularly in Nm, the core metabolism was shown a key player in colonization and development of invasive diseases. In ex vivo transcriptomic analyses, genes encoding enzymes involved in glycolysis, such as Pgm and TpiA; Icd of the TCA cycle, and the majority of the NADH:ubiquinone oxidoreductase subunits were upregulated upon incubation of $\mathrm{Nm}$ cells in human blood $[48,53,63]$. $d p r A$ was also identified as one of the 73 genes required by $\mathrm{Nm}$ to develop septicemic disease in an infant rat model [28]. Electron transport chain-defective Staphylococcus aureus was reported to produce small colony variants highly sensitive to ROS produced by the host immune cells [62]. At the same time, proteins of the amino acid metabolism (ArgF and $\mathrm{ArgH}$ ), glycolysis (GapA-2), and tricarboxylic acid (TCA) cycle (NqrB) were more abundant, which might account for compensatory mechanisms.

The c-type cytochromes are required in the final stage of the electron transport chain that is, they mediate the transfer of electrons from reduced cytochrome c to oxygen $[64,65]$. Simultaneously, the c-type cytochromes contribute to the production of small amounts of superoxide anion and hydrogen peroxide [66]. The abundances of the c-type cytochromes (cytochrome c, cytochrome c5, and PetC) were all reduced in the lack of DprA. Nm MC58 mutants of the cytochromes 44 and putative cytochrome c (NMB0717) grow poorly under aerobic condition [64]. Previously, petC and cytochrome c5 (NMB1677) were shown to be upregulated in $\mathrm{Nm}$ grown in human 
blood $[48,53]$. Nm cytochromes are important for growth under aerobic conditions, but also participate in the production of ROS $[64,66]$. The enzymes catalyzing the detoxification of ROS, the catalase (Kat) and thioredoxin reductase (TrxB) were less abundant in $d p r A$ mutant. The Kat-including superoxide dismutases ( $\operatorname{SodB}$ and $\operatorname{SodC}$ ), glutathione peroxidase (GpxA), and disulfide oxidoreductase (DsbA) are among some of the ample Nm defenses against oxidative stress [67]. In Nm incubated with human blood cells, kat and SodC were upregulated [48]. TrxB was also reported to be important in the defense against oxidative- and disulfide-stress in $S$. aureus and $H$. pylori $[58,68]$. In general, considering the absence of the membrane bound malate:quinone oxidoreductases in mammalian mitochondria whereas they are essential for growth [61] and the absence of DprA in mammals, implies that these proteins can be used as a potential drug target against pathogenic bacteria. Furthermore, the influence of the deletion of $d p r A$ on the core metabolism and the defense against oxidative stress indicates the importance of DprA for Nm pathogenesis.

The Nm ABC transporter protein NMB1240 was shown to be essential in causing septicemic disease in an infant rat model [28]. In the $\mathrm{Nm} d p r A$ mutant, $\mathrm{ABC}$ transporter proteins NMB0387, NMB1240, and NMB1226 were significantly less abundant. $A B C$ transporters are transmembrane permeases used for import and export of proteins, amino acids, sugars, ions or drugs in bacteria [69]. Besides, $A B C$ transporters were found to be involved in competence for transformation, for example, the EcsB of B. subtilis [69] and the adc locus encoding an $\mathrm{ABC}$ transporter in S. pneumoniae [70]. Taken together, the results imply that DprA not only is directly involved in bacterial competence for natural transformation, but also acts indirectly with proteins from multiple cellular pathways.

Previous genetic and biochemical studies have shown that DprA is required for transformation; as for RMP, it was also shown that DprA interacts with SSB and the RecA [4, 8, 15-17, 20, 21]. The interaction and expression of some of the RMPs in neisserial transformation has previously been defined [71]. Proteins interact or are connected either by functioning in similar pathways or through direct or indirect regulatory networks.

In studies of genetic interactions, one of the customary approaches to understanding genetic interactions is to observe how individual proteins behave with respect to each other, either under certain environmental stress conditions, or under altered status of the cell which could be deletion of a gene [72, 73]. Proteomics is an important tool for the study of biological systems. The discovery of new diagnostic biomarkers and indirectly pursuing new therapeutic routes is broadly dependent on the discovery of significant dissimilarities/similarities between two cellular states to unravel the cellular and molecular mechanisms involved in a process; in this context, proteins are main targets as they carry out the major portion of cellular functions [74]. In an attempt to develop an effective vaccine against $\mathrm{Nm}$ serogroup $\mathrm{B}$, comparative global proteomics of $\mathrm{Nm}$ serogroup B and the closely related but non-pathogenic $N$. lactamica was conducted to identify potential vaccine candidate proteins common to the two species [75]. Similar proteomic approaches were used in a pursuit for novel molecular therapeutic targets and potential $\mathrm{Ng}$ vaccine antigens $[76,77]$.

Through pull-down experiments, MS and bioinformatics analyses, we demonstrated the global network of mainly inner membrane proteins and pathways affected in the lack of Nm DprA (Figs. 5 and 7). Such an application of proteogenomics on bacterial wildtype and mutant cells represents a useful approach to enhance the output of traditional microbial genetics and bridging subdisciplines of the -omics. Notably, the study suggests involvement of DprA directly or indirectly in Nm virulence together with the competence proteins important for pilus biogenesis and DNA transformation, as well as the recombinase RecA (Fig. 7). In the context of the already well known function of DprA, proteins involved in neisserial transformation through pilus biogenesis (PilG, PilM, PilT1, PilT2, PilE, PilQ, PilF, PilO, and the pilus substrate PilE) and recombination (RecA) were affected by the loss of DprA.

\section{Conclusion}

We confirm that DprA is directly involved in competence for natural transformation via RecA loading and protection of the incoming ssDNA from degradation by restriction endonucleases [15, 78]. The depiction of DprA-related DA proteins, including the proteins that directly interact with DprA in a pull-down assay, also suggests an indirect role for DprA in colonization and pathogenesis by influencing the expression of $\mathrm{Nm}$ pilus biogenesis components as well as core inner membrane proteins that are important for adaptation to stress.

\section{Methods}

\section{Strains and growth conditions}

Nm strains serogroup B MC58 wildtype [79], the $\Delta d p r A$ mutant (MC58 dprA::aph) [80] constructed by Hovland et al. [36], and the MC58 $\Delta$ pilG mutant [43] were grown on GC agar plates, or in $\mathrm{CO}_{2}$-saturated $\mathrm{GC}$ broth at $37{ }^{\circ} \mathrm{C}$ in $5 \% \mathrm{CO}_{2}$. GC plates and broth were supplemented with $1 \%(v / v)$ IsoVitaleX. E. coli strains were grown in LB medium or on LB agar plates at $37^{\circ} \mathrm{C}$. When applicable, antibiotics were used in the following concentrations: $100 \mu \mathrm{g} / \mathrm{ml}$ ampicillin, $50 \mu \mathrm{g} / \mathrm{ml}$ kanamycin, $8 \mu \mathrm{g} / \mathrm{ml}$ erythromycin. 


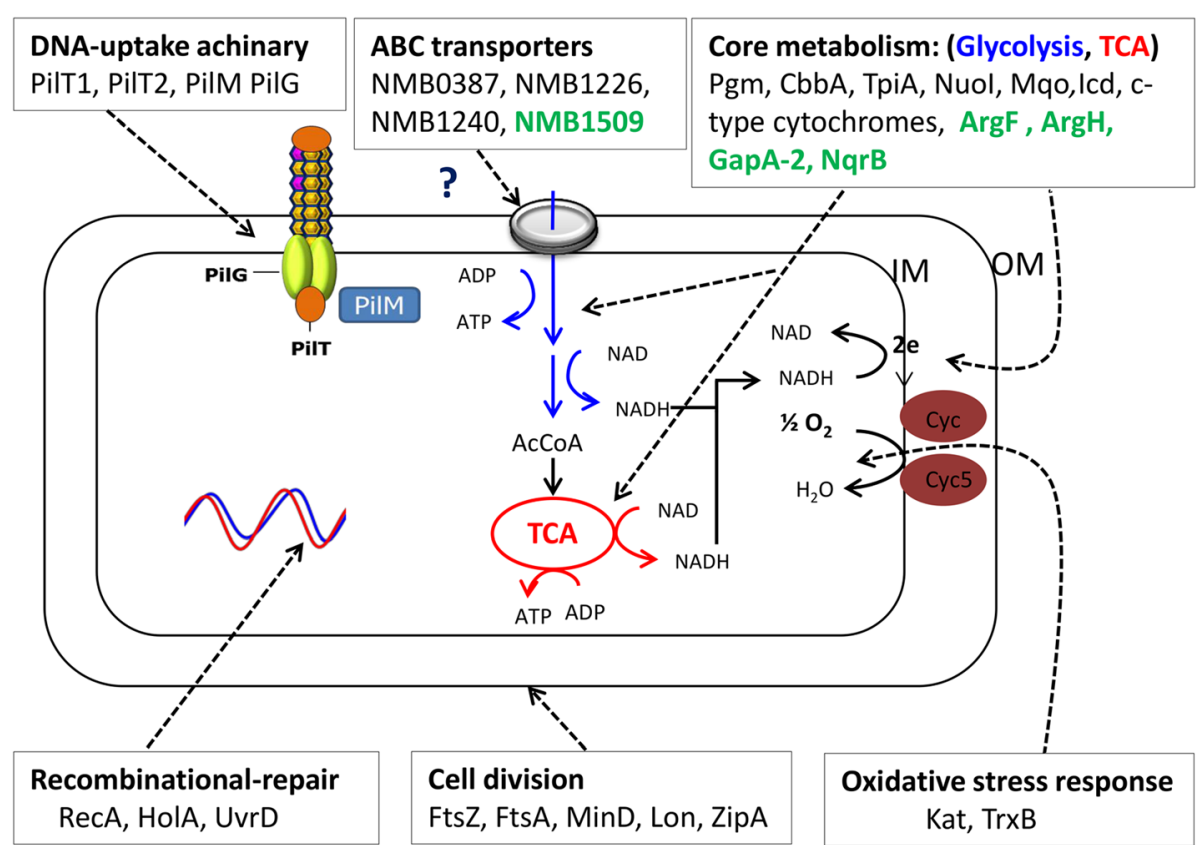

Fig. 7 Schematic graphical presentation of the major pathway components or proteins significantly regulated in the Neisseria meningitidis (Nm) dprA mutant. Downregulated proteins are written in black text and upregulated in green. The figure illustrates: the limitation on the nutrient transport of $\mathrm{Nm}$ from the outside source possibly imposed by the reduced expression of ABC transporters and the subsequent effect on the $\mathrm{Nm}$ metabolism. The enzymes affected within the Nm core metabolism network (glycolysis, TCA cycle and electron transport chain). The less expressed enzymes involved in the production (cytochromes) and detoxification of ROS byproducts of metabolism. Components of the DNA-uptake machinery involved in the internalization of foreign DNA, proteins necessary for the integration of the internalized foreign DNA, DNA damage-repair, and replication, including the proteins required for proper division of the cell that are less expressed in the dprA mutant. IM- inner membrane, OM- outer membrane, CycCytochrome c, Cyc5- Cytochrome c5

\section{Proteomic analysis}

i) Protein extraction. Protein samples were prepared as depicted in Additional file 5: Figure S1. Overnight culture of $\mathrm{Nm}$ wild type and $\Delta d p r A$ mutant cells were harvested into $15 \mathrm{ml}$ tubes (Sarstedt, Germany), washed three times in $1 \times$ PBS buffer, and heat inactivated at $60{ }^{\circ} \mathrm{C}$ for $30 \mathrm{~min}$. The inactivated cell pellets were transferred into Lysing Matrix B tubes (Roche, US), resuspended in 2\% SDS/10 mM Tris- $\mathrm{HCl}, \mathrm{pH} 7.5$ containing EDTA free protease inhibitor cocktail (Roche) and PhosStop (Roche) and mechanically disrupted by bead beating with $\mathrm{MagNa}$ Lyser (Roche, US) at speed for $90 \mathrm{~s}$. The lysis procedure was repeated six times with 1 min cooling on ice in between. The lysate was clarified by centrifugation $(15,000 \times g$ for $15 \mathrm{~min}$ ) at $21^{\circ} \mathrm{C}$. The supernatant containing the lysate proteins were saved and concentration was determined by infrared spectrometry (DirectDetect ${ }^{\mathrm{TM}}$ Spectrometer, Millipore), aliquoted and stored at $-80{ }^{\circ} \mathrm{C}$ until further use. ii) Gel electrophoresis and in-gel digestion. Proteins were separated based on their molecular weight by one-dimensional discontinuous sodium dodecyl sulfate polyacrylamide gel electrophoresis (SDS-PAGE) $(1.0 \mathrm{~mm}$, 4\%-12\% NuPAGE Novex Bis-Tris gel, Invitrogen). A working protein lysate of $100 \mu \mathrm{g}$ was dissolved in $30 \mu \mathrm{l}$ NuPAGE LDS sample buffer $(4 \times)$ and NuPAGE sample reducing agent (10X) (Life Technologies). Samples were heated at $70{ }^{\circ} \mathrm{C}$ for $10 \mathrm{~min}$ before they were loaded onto 10 -well NuPAGE gels. SDS-PAGE was run using $1 \times$ MOPS buffer at $80 \mathrm{~V}$ for $5 \mathrm{~min}$ followed by $20 \mathrm{~min}$ at $200 \mathrm{~V}$. Gels were then stained using a colloidal Coomassie blue staining kit as per the manufacturer's protocol. The entire protein gel lane was cut horizontally into six gel fractions. Gel slices were further cut into smaller pieces and destained at room temperature using 50\% propanol in MQ (ultrapure) water, followed by dehydration in $100 \%$ propanol at $1000 \mathrm{rpm}$ for $1 \mathrm{~h}$.

After destaining, each fraction was subjected to in-gel reduction, alkylation, and trypsin digestion [81]. In brief, protein samples were reduced using $10 \mathrm{mM}$ DTT for $1 \mathrm{~h}$ at $1000 \mathrm{rpm}$ and $56{ }^{\circ} \mathrm{C}$, and alkylated with $55 \mathrm{mM}$ iodoacetamide (IAA) for $1 \mathrm{~h}$ at $1000 \mathrm{rpm}$ and room temperature in dark. Gel fractions were further washed and dehydrated using 50\% and 100\% propanol, respectively, and vacuumdried by Eppendorf 5301 concentrator. Reduced and alkylated samples were then saturated with $50 \mu \mathrm{l}$ trypsin solution of $16.6 \mathrm{ng} / \mu \mathrm{l}$ sequence-grade porcine trypsin (Promega) in $50 \mathrm{mM}$ ammonium bicarbonate. Gel fractions were then incubated at $4{ }^{\circ} \mathrm{C}$ for $2 \mathrm{~h}$ after which protein digestion was achieved by overnight incubation at $37{ }^{\circ} \mathrm{C}$ at $400 \mathrm{rpm}$ [81]. 
The resulting peptide products were sequentially extracted using $100 \mu \mathrm{L}$ of (50\% and $100 \%$ acetonitrile) buffer at room temperature at $600 \mathrm{rpm}$ for $20 \mathrm{~min}$. The supernatant was transferred into new microtubes, concentrated in a vacuum-drier (Eppendorf 5301 concentrator) and resuspended in $30 \mu \mathrm{L}$ of $0.05 \%(\mathrm{v} / \mathrm{v})$ trifluoroacetic acid (TFA). iii) Co-immunoprecipitation (CO-IP) and in-solution tryptic digestion: to proteins that interact with DprA, PilG, and PilE, protein complexes were purified by Co-IP using Abs directed against DprA, PilG, or PilE as per the manufacturer's protocol (Dynabeads Co-Immunopricipitation Kit, Novex life technologies) and analyzed by in-solution tryptic digestion MS. Briefly, the Co-IP purified protein complexes were lyophilized using a centrifugal vacuum concentrator without heat overnight, and solubilized in $30 \mu$ urea buffer (6 M urea in $50 \mathrm{mM}$ ammonium bicarbonate, $\mathrm{ABC}$ ), reduced for $30 \mathrm{~min}$ at room temperature with $1 \mathrm{mM}$ DTT and then alkylated for $15 \mathrm{~min}$ by $5 \mathrm{mM}$ Iodoacetamide. The samples were diluted four fold in $50 \mathrm{mM} \mathrm{ABC}$, digested by trypsin at a ratio of $1: 100 \mathrm{w} / \mathrm{w}$ (trypsin: protein ratio) overnight at $37{ }^{\circ} \mathrm{C}$ with shaking at $400 \mathrm{rpm}$. Subsequently, the peptide products obtained both from in- gel and insolution tryptic digest were desalted on reverse phase $\mathrm{C}_{18}$ Stop-and-Go Extraction (STAGE) tips [82]. The peptide samples were loaded onto ZipTip $\mathrm{C}_{18}$ tips activated and equilibrated with 95\% ACN/0.1\% FA and $0.1 \%$ formic acid (FA), respectively. The loaded peptide products were washed with $0.05 \%$ TFA and eluted with 95\% ACN $/ 0.1 \%$ FA. The eluent was dried using vacuum-drier (Eppendorf 5301 concentrator), dissolved in $0.1 \%$ formic acid (FA) and transferred to autosampler nano-LC vials prior to liquid chromatography tandem mass spectrometry (LC-MS/MS) analysis. iv) Mass spectrometry analysis. Peptide products were analyzed by nano-LC-MS/MS using a Q Exactive hybrid quadropole-orbitrap mass spectrometer interfaced with an EASY-spray ion source (Thermo Fisher Scientific) and coupled to a nano-LC HPLC (Easy nLC1000, Thermo). The peptides were loaded onto a trap column (C18, $100 \mu \mathrm{m} \times 2 \mathrm{~cm}$, PepMap RSLC, Thermo Fisher Scientific) and separated on EASY-Spray columns (PepMapRSLC, C18, $2 \mu \mathrm{m}$ particles, $100 \AA$, $50 \mathrm{~cm}, 75 \mu \mathrm{m}$ ID, Thermo Scientific) using a $2 \mathrm{~h}$ binary gradient as follow: from $2 \%$ to $30 \%$ solvent B in $90 \mathrm{~min}$ followed by $30 \%$ to $45 \%$ solvent B in100-115 min and kept at 90\% B until $120 \mathrm{~min}$ at a flow rate of $0.3 \mu \mathrm{L} / \mathrm{min}$. Solvents used were $0.1 \% \mathrm{FA} / 3 \%$ acetonitrile (solvent $\mathrm{A}$ ) and $0.1 \% \mathrm{FA}$ in $100 \% \mathrm{ACN}$ as solvent $\mathrm{B})$. The column was operated at constant temperature of $60{ }^{\circ} \mathrm{C}$. The LC was coupled to a $\mathrm{Q}$ Exactive mass spectrometer via an Easy nanoelectrospray source (Thermo Scientific). The MS instrument was operated in data dependent acquisition mode with automatic switching between MS and MS/MS scans. Full MS scans were acquired in resolution of 70,000 , with automatic gain control target value of $3 \times 10^{6}$ ions or maximum injection time of $100 \mathrm{~ms}$ within the scan range 400-1200 m/z. Peptide fragmentation was performed by higher energy collision dissociation (HCD) with normalized collision energy set to 25 . The MS/MS spectra were acquired of the 10 most abundant ions (top 10 method) in the resolution $R=17,500$, automatic gain control target value of $1 \times 105$ ions, or maximum fragment accumulation time of $200 \mathrm{~ms}$. An isolation window of $3 \mathrm{Da}$ was used. v) Database searching and analysis. Analysis of MS data was performed using MaxQuant software package (version 1.4.0.5) as described by J Cox and M Mann [83]. Tandem mass spectra (MS/MS) were searched by the Andromeda search engine [84] against the UniProtKB FASTA database for the Nm serogroup B (strain MC58) (2001 entries, downloaded from www.UniProt.org April 2016) using the following parameters: Enzyme specificity was set as Trypsin/P, and a maximum of two missed cleavages and a mass tolerance of $0.5 \mathrm{Da}$ for fragment ion were applied. The 'requantify' and 'match between runs' options were checked with a retention time alignment window of 3 min. Oxidations (M), acetylation (protein N term), Glnpyro (Q) and pyro-Glu (E) were specified as variable modifications and carbamidomethyl $(\mathrm{C})$ as fixed modification.

Database searches were performed with a mass tolerance of $20 \mathrm{ppm}$ for precursor ion for mass calibration, and with a $6 \mathrm{ppm}$ tolerance after calibration. The maximum false peptide and protein discovery rate was specified as 0.01 . Seven amino acids were required as minimum peptide length. Proteins with at least two peptides of which at least one is unique were considered as reliably identified. Following protein identification by a database search, validation for multiple comparisons was corrected using the BenjaminiHochberg correction [85]. To aid in the control of false positives, the database was supplemented with additional sequences for common contaminants and reversed sequence of each entry. The default settings were applied for all other parameters. vi) Statistics and ontology analysis. The statistical determination of protein abundances was assessed using Perseus software (version 1.5.1.6, http://www.perseus-framework.org) as previously described [86]. We used the proteins groups output from MaxQuant as the basis for all the subsequent statistical and ontology enrichment analysis. Intensity based absolute quantification (LFQ) values were used to assess differences in the abundance of proteins between the three biological replicates of Nm wildtype and $\Delta d p r A$ mutant cells. Briefly, the protein groups output was filtered by removing matches to the reverse database, matches only identified by site, and common 
contaminants. Subsequently, LFQ values were transformed to $\log _{2}$. For quantitation, at least two valid LFQ values out of the three biological experiments were required. Signals that were originally zero (missing values) were imputed with random numbers from a normal distribution, whose mean and standard deviation were chosen to best simulate low abundance values below the noise level (width $=0.3$; shift $=1.8$ ) [86].

To identify proteins whose abundance were significantly differentially abundant between the wildtype and $\triangle d p r A$ mutant, a two tailed unpaired $\mathrm{t}$ test was used using a false discovery rate (FDR) value of 0.05 and $\mathrm{S} 0=2$. The S0 parameter sets a threshold for minimum fold change [87]. The resulting significant proteins were analyzed for annotation enrichments. A two-tailed Fisher's exact test was used to assess the significance of enrichment terms. Proteins assigned to enriched term categories ( $p$-value $<0.05)$ were grouped according to the KEGG classification. Vii) Proteins interaction network analysis. To further interpret the biological significance of differentially regulated proteins in terms of protein-protein interaction networks, we used the search tool for the retrieval of interacting genes version 10.0 [88] (STRING, http://string-db.org/) online tool. The required minimum interaction score of at least 0.4 was used as the cut-off criterion. Then, Cytoscape software [89] was used to visualize the interaction network. The properties of the network including node degree and edge attributes were analyzed. Nodes represent proteins and edges represent the interactions/connections between proteins. The degree represents the number of interactions associated with the protein. Proteins with a large degree are known as hub proteins [90] and they are considered to be the essential or key protein in the network [91]. The Network Analyzer option in Cytoscape 3.4.1 was used to compute the degree and betweenness centrality of the network [92].

\section{Immunoblotting}

Whole cell lysates from MC58 were separated by SDSPAGE (Novex), and transferred onto polyvinylidene fluoride (PVDF) membrane. Membranes were washed with Tris buffered saline (TBS) buffer containing 0.05\% $(v / v)$ Tween 20. Blocking was performed with non-fat dry milk. Primary antibody incubation was overnight at $4^{\circ} \mathrm{C}$ with affinity purified rabbit polyclonal antibodies raised against recombinant protein. Secondary antibody incubation with anti-rabbit-IgG-horseradish peroxidase conjugate was performed at room temprature for $4 \mathrm{~h}$. The immunoblots were developed using the Immun-Star WesternC Chemiluminescent kit (Bio-Rad) and visualized using a ChemiDoc Touch imager (Bio-Rad). Results were analysed using the Image Lab software (Bio-Rad).

\section{Co-immunoprecipitation-coupled MS and immunoblotting}

Pull-down experiments by Co-IP and MS analysis was used to further investigate DprA, PilG, and PilE interactions to each other and with other proteins by incubating the cell lysates with polyclonal rabbit Abs raised against DprA and PilG recombinant proteins and purified Tfp (PilE). For this purpose, Nm strains MC58 wildtype, $\triangle d p r A$, and $\Delta$ pilG were grown overnight $(\mathrm{ON})$ on $\mathrm{GC}$ agar plates. The next day, piliated colonies were picked under a stereo-microscope, and uniformly cultured on GC agar plates. From the ON culture, between 1 and $1.2 \mathrm{~g}$ of cells were harvested and washed three times in $1 \%$ phosphate-buffered saline, $\mathrm{pH} 7.4$ (PBS). The cell pellets were lysed by the detergent lysis method according to the manufacturer's protocol (Dynabeads Co-Immunoprecipitation Kit, Novex life technologies) with minor modifications. Briefly, the cell pellets were resuspend in 1:9 ratio of cell mass to extraction buffer [100 mM NaCl, $2 \mathrm{mM} \mathrm{MgCl,} 1 \mathrm{mM}$ DTT, and 1\% n-Dodecyl $\beta$-D-maltoside (DDM)] containing protease inhibitors, and incubated on ice for $15 \mathrm{~min}$. The cell lysates were centrifuged at $5000 \times \mathrm{g}$ for $5 \mathrm{~min}$ at $4{ }^{\circ} \mathrm{C}$, and the supernatants were immediately used for coimmunoprecipitation. Appropriate amount of Dynabeads coupled to anti-DprA or anti-PilG antibodies according to the manufacturer's protocol were added to the cell lysates, and incubated on a rotator at $4{ }^{\circ} \mathrm{C}$ for $1.5 \mathrm{~h}$. The incubation allowed DprA or PilG proteins to bind their respective antibody-coupled Dynabeads; in turn, DprA or PilG bound their interacting protein partners within the cell lysate, yielding protein complexes. Finally, the Dynabeads were washed, and the protein complexes were eluted by the elution buffer supplied with the kit. The eluted protein complex samples were analyzed by MS (as described in proteomic analysis sections iii-v), and immunoblotting as described above, except that the immunoblots were detected using 5-bromo-4-chloro-39-indolyl phosphate p-toluidine salt (BCIP) and nitro-blue tetrazolium chloride (NBT) as substrates for the AP [41].

\section{Additional files}

Additional file 1: Table S1. Complete list of all the significantly differentially abundant proteins identified by mass spectrometry. (PDF 152 kb)

Additional file 2: Figure S2. PilG and DprA expression is reduced in $\mathrm{Nm} \triangle d p r A$ and $\triangle p i l G$ mutants, respectively. A) Representative gel images of the $\triangle d p r A$ mutant-, and B) $\Delta p i l G$ mutant-, compared to the wild type $\mathrm{Nm}$ as analysed by western blot. In both $\mathrm{A}$ and $\mathrm{B}$ lanes $1-4$ represent 0.625 , $1.25,2.5$ and $5 \mu \mathrm{g}$ of cell lysates from Nm wild type, Lane 5 in A and B are negative controls that is, cell lysates from $\triangle p i l G$ and $\triangle d p r A$, respectively. In both $A$ and $B$ lane $6-9$ contains $0.625,1.25,2.5$ and $5 \mu \mathrm{g}$ of cell lysates from the corresponding $\mathrm{Nm}$ knockout mutant strains. (TIFF $931 \mathrm{~kb}$ ) 
Additional file 3: Figure S3. DprA interacts with PilG. A) PilG COimmunoprecipitated using anti-DprA antibody from the cell lysates, and confirmed by western blot using anti-PilG antibody. Cell lysates samples indicated by lane 1, 2, and 3 were from the Nm MC58 wild type, $\triangle d p r A$, and $\triangle$ pilG, respectively). Also, the presence of PilG band at lane 2 suggests the direct interaction and co-immunoprecipitation of anti-DprA antibody with PilG in the absence of DprA. B) Western blot by anti-PilE antibody showing that PilE expression of the $\mathrm{Nm} \triangle d p r A$ mutant is comparable with the wild type $\mathrm{Nm}$ PilE expression; 20 and $40 \mu \mathrm{g}$ cell lysates were used from each sample; lanes 1-2, 3-4, and 5-6 indicate lysates from Nm wild type, $\triangle d p r A$, and $\triangle p i l G$, respectively. The band corresponding to PilE is absent in $\triangle$ pilG mutant Nm, lane 5 and 6. C) Single point quantitation of PilE western blot using Image Studio Lite analysis software. (TIFF $759 \mathrm{~kb}$ )

Additional file 4: Table S2. Proteins identified interact with DprA, PilG, or PilE by Co-Immunopricipitation (Co-IP). During CO-IP DprA, PilG, or PilE was used as bait proteins. In order for DprA, PilG or PilE target their interacting proteins, the antibody (Ab) against the bait proteins were incubated with the cell lysates from $\mathrm{Nm}$ wild type $(\mathrm{Wt})$, also incubated with the cell lysates from $\triangle d p r A$, or $\triangle$ pilG mutant $\mathrm{Nm}$ (in the mutant the bait protein is absent), subsequently the antibody bind the bait protein. The bait protein coupled with the antibody binds its interacting partner, and form antibody-bait-prey protein complex. The " + " sign designates the formation of antibody-bait-prey protein complex whereas the "-" sign designates the absence of complex formation/interaction. (PDF $256 \mathrm{~kb}$ )

Additional file 5: Figure S1. Schematic diagram of the proteomics work flow. MC58 wt and $\triangle d p r A$ mutant overnight (ON) cultures were harvested, washed $3 \times$ with $1 \times$ PBS, centrifuged at 6000 RPM for 10 min. The pellets were resuspend in lysis buffer, transferred into Lysing Matrix B tubes containing $0.1 \mathrm{~mm}$ silica beads, and disrupted using MagNa Lyser (6x 90s at a speed of 6000). The cell lysates cleared by spinning down at 15,000 xg for $15 \mathrm{~min}$, and the supernatant (containing protein) were analyzed by SDS-PAGE. The gel lanes were cut into six pieces, and digested with trypsin. The peptides products were extracted and purified, and injected into an electrospray-based Q-Exactive MS. The MS output proteins were identified and quantified using MaxQuant as described in method section. Finally, the differentially expressed proteins were functional annotated (KEGG). (TIFF 2976 kb)

\section{Abbreviations}

ABC: ATP-binding cassette; Co-IP: co-immunoprecipitation; DA: Differentially abundant; DprA: DNA processing chain A; KEGG: Kyoto Encyclopedia of Genes and Genomes; LFQ: Label free quantitative; MS: Mass spectrometry; NAD: Nicotinamide adenine dinucleotide; Nm: Neisseria meningitidis; ROS: reactive oxygen species; SSB: single-strand binding protein; TCA: the tricarboxylic acid cycle

\section{Acknowledgements}

We appreciate the technical assistance from Anoe Gelen in preparing the MS material from the Nm $\triangle$ pilG mutant. We also thank Håvard Homberset for reading the manuscript and providing invaluable comments.

\section{Funding}

This work was supported by the Research Council of Norway (RCN) (GLOBVAC project \#220901 to TT), the University of Oslo Quota program / Norwegian State Educational Loan Fund (GTB) and Mekelle University (GTB). The funders did not have any role in the design of the study and collection, analysis, and interpretation of data and in writing the manuscript.

\section{Availability of data and materials}

The mass spectrometry proteomics data have been deposited to the ProteomeXchange Consortium via the PRIDE [93] partner repository with the dataset identifier PXD00612. The data can be accessed using the username: reviewer90340@ebi.ac.uk and password: zEcQOQsB.

\section{Authors' contributions}

TT \& GTB conceived the study and study design. GTB and TT performed strain handling and cultivation. GTB and SK performed protein/peptide sample preparation. SK and TR performed the MS analysis. SK, GTB and TR performed the bioinformatics and statistical analyses. GTB performed the coimmunoprecipitation and immunoblotting. GTB, TT, SK, and TR evaluated and interpreted the data and drafted the paper. All authors approved the final manuscript.

\section{Competing interests}

The authors declare that they have no competing interests.

\section{Consent for publication}

Not applicable.

\section{Ethics approval and consent to participate}

The serogroup B MC58 wildtype strain was retrieved from the World Health Organization meningococcal strain collection, originally isolated in the UK by McGuinness et al. in 1991 [93]. The MC58 $\triangle$ dprA and $\Delta$ pilG mutant strains were derived from the MC58 wildtype strain. We have authorized biosafety level-2 laboratory space for handling meningococcal strains, and the permission for this use has formally been granted.

\section{Publisher's Note}

Springer Nature remains neutral with regard to jurisdictional claims in published maps and institutional affiliations.

\section{Author details}

'Department of Microbiology, University of Oslo, Oslo, Norway. ${ }^{2}$ Department of Microbiology, Oslo University Hospital, Oslo, Norway. ${ }^{3}$ Mekelle University College of Veterinary Medicine, Mekelle, Ethiopia. ${ }^{4}$ Present address: College of Health Sciences, Mekelle University, Mekelle, Ethiopia.

Received: 23 December 2016 Accepted: 8 April 2017

Published online: 21 April 2017

\section{References}

1. Stephens DS, Greenwood B, Brandtzaeg P. Epidemic meningitis, meningococcaemia, and Neisseria meningitidis. Lancet. 2007;369(9580):2196-210.

2. Davidsen T, Tønjum T. Meningococcal genome dynamics. Nat Rev Microbiol. 2006:4(1):11-22.

3. Chen I, Christie PJ, Dubnau D. The ins and outs of DNA transfer in bacteria. Science. 2005:310(5753):1456-60.

4. Sun YH, Exley R, Li Y, Goulding D, Tang C. Identification and characterization of genes required for competence in Neisseria meningitidis. J Bacteriol. 2005; 187(9):3273-6.

5. Caugant DA. Genetics and evolution of Neisseria meningitidis: importance for the epidemiology of meningococcal disease. Infect Genet Evol. 2008;8(5):558-65.

6. Berry $J-L$, Cehovin A, McDowell MA, Lea SM, Pelicic V. Functional analysis of the interdependence between DNA uptake sequence and its cognate ComP receptor during natural transformation in Neisseria species. PLoS Genet. 2013:9(12):e1004014.

7. Mortier-Barriere I, de Saizieu A, Claverys JP, Martin B. Competence-specific induction of recA is required for full recombination proficiency during transformation in Streptococcus pneumoniae. Mol Microbiol. 1998;27(1):159-70.

8. Berge M, Mortier-Barriere I, Martin B, Claverys JP. Transformation of Streptococcus pneumoniae relies on DprA- and RecA-dependent protection of incoming DNA single strands. Mol Microbiol. 2003:50(2):527-36.

9. Beernink HT, Morrical SW. RMPs: recombination/replication mediator proteins. Trends Biochem Sci. 1999;24(10):385-9.

10. Koomey JM, Falkow S. Cloning of the recA gene of Neisseria gonorrhoeae and construction of gonococcal recA mutants. J Bacteriol. 1987;169(2):790-5.

11. Attaiech L, Olivier A, Mortier-Barriere I, Soulet AL, Granadel C, Martin B, Polard P, Claverys JP. Role of the single-stranded DNA-binding protein SsbB in pneumococcal transformation: maintenance of a reservoir for genetic plasticity. PLoS Genet. 2011;7(6):e1002156.

12. Yadav T, Carrasco B, Myers AR, George NP, Keck JL, Alonso JC. Genetic recombination in Bacillus subtilis: a division of labor between two singlestrand DNA-binding proteins. Nucleic Acids Res. 2012;40(12):5546-59.

13. Yadav T, Carrasco B, Serrano E, Alonso JC. Roles of Bacillus subtilis DprA and SsbA in RecA-mediated genetic recombination. J Biol Chem. 2014;289(40):27640-52.

14. Chen Z, Yang H, Pavletich NP. Mechanism of homologous recombination from the RecA-ssDNA/dsDNA structures. Nature. 2008;453(7194):489-4.

15. Mortier-Barriere I, Velten M, Dupaigne $P$, Mirouze N, Pietrement $O$ McGovern S, Fichant G, Martin B, Noirot P, Le Cam E, et al. A key presynaptic role in transformation for a widespread bacterial protein: DprA conveys incoming ssDNA to RecA. Cell. 2007;130(5):824-36. 
16. Quevillon-Cheruel S, Campo N, Mirouze N, Mortier-Barriere I, Brooks MA, Boudes M, Durand D, Soulet AL, Lisboa J, Noirot P, et al. Structure-function analysis of pneumococcal DprA protein reveals that dimerization is crucial for loading RecA recombinase onto DNA during transformation. Proc Natl Acad Sci U S A. 2012;109(37):E2466-75.

17. Yadav T, Carrasco B, Hejna J, Suzuki Y, Takeyasu K, Alonso JC. Bacillus subtilis DprA recruits RecA onto single-stranded DNA and mediates annealing of complementary strands coated by SsbB and SsbA. J Biol Chem. 2013;288(31):22437-50

18. Dwivedi GR, Sharma E, Rao DN. Helicobacter pylori DprA alleviates restriction barrier for incoming DNA. Nucleic Acids Res. 2013;41(5):3274-88.

19. Duffin PM, Barber DA. DprA is required for natural transformation and affects pilin variation in Neisseria gonorrhoeae. Microbiology. 2016;162(9):1620-8.

20. Karudapuram S, Zhao X, Barcak GJ. DNA sequence and characterization of Haemophilus influenzae dprAt, a gene required for chromosomal but not plasmid DNA transformation. J Bacteriol. 1995;177(11):3235-40.

21. Tadesse S, Graumann PL. DprA/Smf protein localizes at the DNA uptake machinery in competent Bacillus subtilis cells. BMC Microbiol. 2007:7:105

22. Weng $L$, Piotrowski A, Morrison DA. Exit from competence for genetic transformation in Streptococcus pneumoniae is regulated at multiple levels. PLoS One. 2013;8(5):e64197.

23. Mirouze N, Berge MA, Soulet AL, Mortier-Barriere I, Quentin Y, Fichant G, Granadel C, Noirot-Gros MF, Noirot P, Polard P, et al. Direct involvement of DprA, the transformation-dedicated RecA loader, in the shut-off of pneumococcal competence. Proc Natl Acad Sci U S A. 2013;110(11):E1035-44.

24. Kramer N, Hahn J, Dubnau D. Multiple interactions among the competence proteins of Bacillus subtilis. Mol Microbiol. 2007;65(2):454-64.

25. Dyet K, Moir J. Effect of combined oxidative and nitrosative stress on Neisseria meningitidis. Biochem Soc Trans. 2006;34(Pt 1):197-9.

26. Tonjum T, Freitag NE, Namork E, Koomey M. Identification and characterization of pilG, a highly conserved pilus-assembly gene in pathogenic Neisseria. Mol Microbiol. 1995;16(3):451-64.

27. Johnston C, Martin B, Fichant G, Polard P, Claverys JP. Bacterial transformation: distribution, shared mechanisms and divergent control. Nat Rev Microbiol. 2014;12(3):181-96.

28. Sun YH, Bakshi S, Chalmers R, Tang CM. Functional genomics of Neisseria meningitidis pathogenesis. Nat Med. 2000:6(11):1269-73.

29. Olczak AA, Olson JW, Maier RJ. Oxidative-stress resistance mutants of Helicobacter pylori. J Bacteriol. 2002;184(12):3186-93.

30. Wong SL, Roth FP. Transcriptional compensation for gene loss plays a minor role in maintaining genetic robustness in Saccharomyces cerevisiae. Genetics. 2005;171(2):829-33.

31. Cox MM. Motoring along with the bacterial RecA protein. Nat Rev Mol Cell Biol. 2007;8(2):127-38.

32. Khanduja JS, Muniyappa K. Functional analysis of DNA replication fork reversal catalyzed by Mycobacterium tuberculosis RuvAB proteins. J Biol Chem. 2012;287(2):1345-60.

33. Kang J, Blaser MJ. UvrD helicase suppresses recombination and DNA damageinduced deletions. J Bacteriol. 2006;188(15):5450-9.

34. Carter AS, Tahmaseb K, Compton SA, Matson SW. Resolving Holliday junctions with Escherichia coli UvrD helicase. J Biol Chem. 2012;287(11):8126-34.

35. Bentchikou E, Chagneau C, Long E, Matelot M, Allemand JF, Michel B. Are the SSB-interacting proteins $\mathrm{RecO}$, RecG, PriA and the DnaB-interacting protein rep bound to progressing replication forks in Escherichia coli? PLoS One. 2015;10(8):e0134892.

36. Hovland $\mathrm{E}$, Frye SA, Homberset $\mathrm{H}$, Tønjum $\mathrm{T}$, Ambur OH. The role of DprA in meningococcal transformation in: 17th IPNC Conference Banff, Canada. In: The Banff centere; 2010. p. 117

37. Crozat E, Rousseau P, Fournes F, Cornet F. The FtsK family of DNA translocases finds the ends of circles. J Mol Microbiol Biotechnol. 2014;24(5-6):396-408.

38. Liu IF, Sutherland JH, Cheng B, Tse-Dinh YC. Topoisomerase I function during Escherichia coli response to antibiotics and stress enhances cell killing from stabilization of its cleavage complex. J Antimicrob Chemother. 2011;66(7):1518-24.

39. Levine C, Hiasa H, Marians KJ. DNA gyrase and topoisomerase IV: biochemical activities, physiological roles during chromosome replication, and drug sensitivities. Biochim Biophys Acta. 1998:1400(1-3):29-43.

40. Frye SA, Lang E, Beyene GT, Balasingham SV, Homberset H, Rowe AD, Ambur $\mathrm{OH}$, Tonjum $\mathrm{T}$. The inner membrane protein PilG interacts with DNA and the Secretin PilQ in transformation. PLoS One. 2015;10(8):e0134954.
41. Lång $E$, Haugen $K$, Fleckenstein $B$, Homberset $H$, Frye SA, Ambur OH, Tønjum T. Identification of neisserial DNA binding components. Microbiology. 2009; 155(Pt 3):852-62.

42. Tonjum T, Caugant DA, Dunham SA, Koomey M. Structure and function of repetitive sequence elements associated with a highly polymorphic domain of the Neisseria meningitidis PilQ protein. Mol Microbiol. 1998;29(1):111-24.

43. Tønjum T, Freitag NE, Namork E, Koomey M. Identification and characterization of pilG, a highly conserved pilus-assembly gene in pathogenic Neisseria. Mol Microbiol. 1995;16(3):451-64.

44. Wolfgang M, Lauer P, Park H-S, Brossay L, Hebert J, Koomey M. PilT mutations lead to simultaneous defects in competence for natural transformation and twitching motility in piliated Neisseria gonorrhoeae. Mol Microbiol. 1998;29(1): $321-30$

45. Seifert HS. Insertionally inactivated and inducible recA alleles for use in Neisseria. Gene. 1997:188(2):215-20.

46. Rumszauer J, Schwarzenlander C, Averhoff B. Identification, subcellular localization and functional interactions of PilMNOWQ and PilA4 involved in transformation competency and pilus biogenesis in the thermophilic bacterium Thermus thermophilus HB27. FEBS J. 2006:273(14):3261-72.

47. Karuppiah V, Derrick JP. Structure of the PilM-PilN inner membrane type IV pilus biogenesis complex from Thermus thermophilus. J Biol Chem. 2011; 286(27):24434-42.

48. Echenique-Rivera H, Muzzi A, Del Tordello E, Seib KL, Francois P, Rappuoli R, Pizza M, Serruto D. Transcriptome analysis of Neisseria meningitidis in human whole blood and mutagenesis studies identify virulence factors involved in blood survival. PLoS Pathog. 2011;7(5):e1002027.

49. Scheuerpflug I, Rudel T, Ryll R, Pandit J, Meyer TF. Roles of PilC and PilE proteins in pilus-mediated adherence of Neisseria gonorrhoeae and Neisseria meningitidis to human erythrocytes and endothelial and epithelial cells. Infect Immun. 1999:67(2):834-43.

50. Miller F, Phan G, Brissac T, Bouchiat C, Lioux G, Nassif X, Coureuil M. The hypervariable region of meningococcal major pilin PilE controls the host cell response via antigenic variation. mBio. 2014;5(1):e01024-13.

51. Freitag NE, Seifert HS, Koomey M. Characterization of the pilF-pilD pilusassembly locus of Neisseria gonorrhoeae. Mol Microbiol. 1995;16(3):575-86.

52. Tammam S, Sampaleanu LM, Koo J, Sundaram P, Ayers M, Chong PA, Forman-Kay JD, Burrows LL, Howell PL. Characterization of the PilN, PilO and PilP type IVa pilus subcomplex. Mol Microbiol. 2011;82(6):1496-514.

53. Hedman AK, Li MS, Langford PR, Kroll JS. Transcriptional profiling of serogroup B Neisseria meningitidis growing in human blood: an approach to vaccine antigen discovery. PLoS One. 2012;7(6):e39718.

54. Pazos M, Natale P, Vicente M. A specific role for the ZipA protein in cell division: stabilization of the FtsZ protein. J Biol Chem. 2013;288(5):3219-26.

55. Sakai N, Yao M, Itou H, Watanabe N, Yumoto F, Tanokura M, Tanaka I. The three-dimensional structure of septum site-determining protein MinD from Pyrococcus horikoshii OT3 in complex with mg-ADP. Structure. 2001;9(9):817-26.

56. Fu GK, Smith MJ, Markovitz DM. Bacterial protease Lon is a site-specific DNA-binding protein. J Biol Chem. 1997;272(1):534-8.

57. Sainsbury S, Ren J, Nettleship JE, Saunders NJ, Stuart DI, Owens RJ. The structure of a reduced form of OxyR from Neisseria meningitidis. BMC Struct Biol. 2010;10:10.

58. Wang G, Maier RJ. An NADPH quinone reductase of Helicobacter pylori plays an important role in oxidative stress resistance and host colonization. Infect Immun. 2004;72(3):1391-6.

59. Kavanagh $\mathrm{KL}$, Jornvall $H$, Persson $\mathrm{B}$, Oppermann U. Medium- and shortchain dehydrogenase/reductase gene and protein families : the SDR superfamily: functional and structural diversity within a family of metabolic and regulatory enzymes. Cellular and molecular life sciences: CMLS. 2008; 65(24):3895-906

60. Pfenninger-Li XD, Albracht SP, van Belzen R, Dimroth P. NADH:ubiquinone oxidoreductase of Vibrio alginolyticus: purification, properties, and reconstitution of the Na+ pump. Biochemistry. 1996;35(20):6233-42.

61. Kabashima Y, Sone N, Kusumoto T, Sakamoto J. Purification and characterization of malate:quinone oxidoreductase from thermophilic Bacillus sp. PS3. J Bioenerg Biomembr. 2013;45(1-2):131-6

62. Painter KL, Strange E, Parkhill J, Bamford KB, Armstrong-James D, Edwards AM. Staphylococcus aureus Adapts to oxidative stress by producing $\mathrm{H}_{2} \mathrm{O} 2-$ resistant small-colony variants via the SOS response. Infect Immun. 2015; 83(5):1830-44

63. Schoen C, Kischkies L, Elias J, Ampattu BJ. Metabolism and virulence in Neisseria meningitidis. Front Cell Infect Microbiol. 2014;4:114. 
64. Deeudom M, Koomey M, Moir JW. Roles of c-type cytochromes in respiration in Neisseria meningitidis. Microbiology. 2008;154(Pt 9):2857-64.

65. Ludwig B, Schatz G. A two-subunit cytochrome c oxidase (cytochrome aa3) from Paracoccus dentrificans. Proc Natl Acad Sci U S A. 1980;77(1):196-200.

66. Berg JM TJ, Stryer L: Biochemistry 5th edition. Section 18.3, The Respiratory Chain Consists of Four Complexes: Three Proton Pumps and a Physical Link to the Citric Acid Cycle. https://www.ncbi.nlm.nih.gov/books/NBK22505/, vol. Section 18.3, 5 edn. New York: W H Freeman 2002.

67. Seib KL, Tseng HJ, McEwan AG, Apicella MA, Jennings MP. Defenses against oxidative stress in Neisseria gonorrhoeae and Neisseria meningitidis: distinctive systems for different lifestyles. J Infect Dis. 2004;190(1):136-47.

68. Ballal A, Manna AC. Control of thioredoxin reductase gene (trxB) transcription by SarA in Staphylococcus aureus. J Bacteriol. 2010;192(1):336-45.

69. Takeno M, Taguchi H, Akamatsu T. Essential involvement of the Bacillus subtilis $A B C$ transporter, EcsB, in genetic transformation of purified DNA but not native DNA from protoplast lysates. J Biosci Bioeng. 2011;112(3):209-14.

70. Dintilhac A, Claverys JP. The adc locus, which affects competence for genetic transformation in Streptococcus pneumoniae, encodes an ABC transporter with a putative lipoprotein homologous to a family of streptococcal adhesins. Res Microbiol. 1997;148(2):119-31

71. Mehr IJ, Seifert HS. Differential roles of homologous recombination pathways in Neisseria gonorrhoeae pilin antigenic variation, DNA transformation and DNA repair. Mol Microbiol. 1998;30(4):697-710.

72. Bandyopadhyay S, Mehta M, Kuo D, Sung MK, Chuang R, Jaehnig EJ, Bodenmiller B, Licon K, Copeland W, Shales M, et al. Rewiring of genetic networks in response to DNA damage. Science. 2010;330(6009):1385-9.

73. Tong AH, Lesage $G$, Bader GD, Ding H, Xu H, Xin X, Young J, Berriz GF, Brost RL, Chang $M$, et al. Global mapping of the yeast genetic interaction network. Science. 2004;303(5659):808-13.

74. Deracinois B, Flahaut C, Duban-Deweer S, Karamanos Y. Comparative and quantitative global proteomics approaches: an overview. Proteomes. 2013; 1(3):180-218.

75. Sheikhi R, Amin M, Hamidinia M, Assarehzadegan MA, Rostami S, Mojtahedi Z. Comparative proteomics analysis of two strains of Neisseria meningitidis Serogroup B and Neisseria lactamica. Jundishapur journal of microbiology. 2015;8(11):e25228.

76. Zielke RA, Wierzbicki $I H$, Baarda BI, Gafken PR, Soge OO, Holmes KK, Jerse AE, Unemo M, Sikora AE. Proteomics-driven antigen discovery for development of vaccines against gonorrhea. Molecular \& cellular proteomics : MCP. 2016;15(7): 2338-55.

77. Zielke RA, Wierzbicki IH, Weber JV, Gafken PR, Sikora AE. Quantitative proteomics of the Neisseria gonorrhoeae cell envelope and membrane vesicles for the discovery of potential therapeutic targets. Molecular \& cellular proteomics : MCP. 2014;13(5):1299-317.

78. Lisboa J, Andreani J, Sanchez D, Boudes M, Collinet B, Liger D, van Tilbeurgh H, Guerois R, Quevillon-Cheruel S. Molecular determinants of the DprA-RecA interaction for nucleation on ssDNA. Nucleic Acids Res. 2014:42(11):7395-408.

79. Tettelin H, Saunders NJ, Heidelberg J, Jeffries AC, Nelson KE, Eisen JA, Ketchum KA, Hood DW, Peden JF, Dodson RJ, et al. Complete genome sequence of Neisseria meningitidis serogroup B strain MC58. Science. 2000; 287(5459):1809-15.

80. Pansegrau W, Miele L, Lurz R, Lanka E. Nucleotide sequence of the kanamycin resistance determinant of plasmid RP4: homology to other aminoglycoside 3'-phosphotransferases. Plasmid. 1987;18(3):193-204.

81. Shevchenko A, Tomas H, Havlis J, Olsen JV, Mann M. In-gel digestion for mass spectrometric characterization of proteins and proteomes. Nat Protoc. 2006;1(6):2856-60.

82. Rappsilber J, Ishihama Y, Mann M. Stop and go extraction tips for matrixassisted laser desorption/ionization, nanoelectrospray, and LC/MS sample pretreatment in proteomics. Anal Chem. 2003;75(3):663-70.

83. Cox J, Mann M. MaxQuant enables high peptide identification rates, individualized p.P.B.-range mass accuracies and proteome-wide protein quantification. Nat Biotechnol. 2008;26(12):1367-72.

84. Cox J, Neuhauser N, Michalski A, Scheltema RA, Olsen JV, Mann M. Andromeda: a peptide search engine integrated into the MaxQuant environment. J Proteome Res. 2011;10(4):1794-805.

85. Benjamini Y, Drai D, Elmer G, Kafkafi N, Golani I. Controlling the false discovery rate in behavior genetics research. Behav Brain Res. 2001;125(1-2):279-84.

86. Hubner NC, Bird AW, Cox J, Splettstoesser B, Bandilla P, Poser I, Hyman A, Mann M. Quantitative proteomics combined with BAC TransgeneOmics reveals in vivo protein interactions. J Cell Biol. 2010;189(4):739-54.
87. Tusher VG, Tibshirani R, Chu G. Significance analysis of microarrays applied to the ionizing radiation response. Proc Natl Acad Sci U S A. 2001;98(9): 5116-21.

88. Szklarczyk D, Franceschini A, Wyder S, Forslund K, Heller D, Huerta-Cepas J, Simonovic M, Roth A, Santos A, Tsafou KP, et al. STRING v10: protein-protein interaction networks, integrated over the tree of life. Nucleic Acids Res. 2015:43(Database issue):D447-52

89. Shannon P, Markiel A, Ozier O, Baliga NS, Wang JT, Ramage D, Amin N, Schwikowski B, Ideker T. Cytoscape: a software environment for integrated models of biomolecular interaction networks. Genome Res. 2003;13(11): 2498-504.

90. Azuaje F, Devaux Y, Wagner DR. Coordinated modular functionality and prognostic potential of a heart failure biomarker-driven interaction network BMC Syst Biol. 2010;4:60.

91. Ideker T, Sharan R. Protein networks in disease. Genome Res. 2008:18(4):644-52.

92. Assenov Y, Ramirez F, Schelhorn SE, Lengauer T, Albrecht M. Computing topological parameters of biological networks. Bioinformatics. 2008;24(2):282-4.

93. Vizcaino JA, Csordas a, del-Toro N, Dianes JA, Griss J, Lavidas I, Mayer G, Perez-Riverol Y, Reisinger F, Ternent T, et al. 2016 update of the PRIDE database and its related tools. Nucleic Acids Res. 2016:44(D1):D447-56.

\section{Submit your next manuscript to BioMed Central and we will help you at every step:}

- We accept pre-submission inquiries

- Our selector tool helps you to find the most relevant journal

- We provide round the clock customer support

- Convenient online submission

- Thorough peer review

- Inclusion in PubMed and all major indexing services

- Maximum visibility for your research

Submit your manuscript at www.biomedcentral.com/submit
) Biomed Central 